\title{
A FAST, SIMPLE, AND STABLE CHEBYSHEV-LEGENDRE TRANSFORM USING AN ASYMPTOTIC FORMULA
}

\author{
NICHOLAS HALE* AND ALEX TOWNSEND ${ }^{\dagger}$
}

\begin{abstract}
A fast, simple, and numerically stable transform for converting between Legendre and Chebyshev coefficients of a degree $N$ polynomial in $\mathcal{O}\left(N(\log N)^{2} / \log \log N\right)$ operations is derived. The basis of the algorithm is to rewrite a well-known asymptotic formula for Legendre polynomials of large degree as a weighted linear combination of Chebyshev polynomials, which can then be evaluated by using the discrete cosine transform. Numerical results are provided to demonstrate the efficiency and numerical stability. Since the algorithm evaluates a Legendre expansion at an $N+1$ Chebyshev grid as an intermediate step, it also provides a fast transform between Legendre coefficients and values on a Chebyshev grid.
\end{abstract}

Key words. Chebyshev, Legendre, transform, asymptotic formula, discrete cosine transform

AMS subject classifications. 65T50, 65D05, 41A60

1. Introduction. Expansions of functions as finite series of orthogonal polynomials have applications throughout scientific computing, engineering, and physics $[4,8,27]$. Expansions in Chebyshev polynomials,

$$
p_{N}(x)=\sum_{n=0}^{N} c_{n}^{c h e b} T_{n}(x), \quad x \in[-1,1]
$$

where $T_{n}(x)=\cos \left(n \cos ^{-1}(x)\right)$, are often used because of their near-optimal approximation properties and associated fast algorithms [17,33]. However, in some situations Legendre expansions,

$$
p_{N}(x)=\sum_{n=0}^{N} c_{n}^{l e g} P_{n}(x), \quad x \in[-1,1],
$$

where $P_{n}(x)$ is the degree $n$ Legendre polynomial, are preferred due to their orthogonality in the standard $L^{2}$ inner product, more rapidly decaying Cauchy transform [20], or connection to spherical harmonics [26].

Unfortunately, fast algorithms are not as readily available for computing with Legendre expansions, and hence a fast transform to convert between Legendre and Chebyshev coefficients is desirable. In this paper we describe a fast, simple, and stable transform that converts between the coefficients $c_{0}^{\text {leg }}, \ldots, c_{N}^{\text {leg }}$ in (1.2) and the coefficients $c_{0}^{c h e b}, \ldots, c_{N}^{c h e b}$ in $(1.1)$ in $\mathcal{O}\left(N(\log N)^{2} / \log \log N\right)$ operations:

$$
c_{0}^{l e g}, \ldots, c_{N}^{\text {leg }} \quad \frac{\text { forward transform }}{\mathcal{O}\left(N(\log N)^{2} / \log \log N\right)} \quad c_{0}^{\text {cheb }}, \ldots, c_{N}^{\text {cheb }} .
$$

Whilst there are a number of existing fast algorithms for computing such transforms, many of these require hierarchical data structures and expensive initialization procedures $[2,25]$, needs an underlying function to evaluate $[7,16]$, or suffer from stability problems [19].

*University of Oxford Mathematical Institute, Oxford, OX26GG, UK. (hale@maths.ox.ac.uk, http://people.maths.ox.ac.uk/hale/).

${ }^{\dagger}$ University of Oxford Mathematical Institute, Oxford, OX2 6GG, UK. (townsend@maths.ox.ac.uk, http://people.maths.ox.ac.uk/townsend/). 


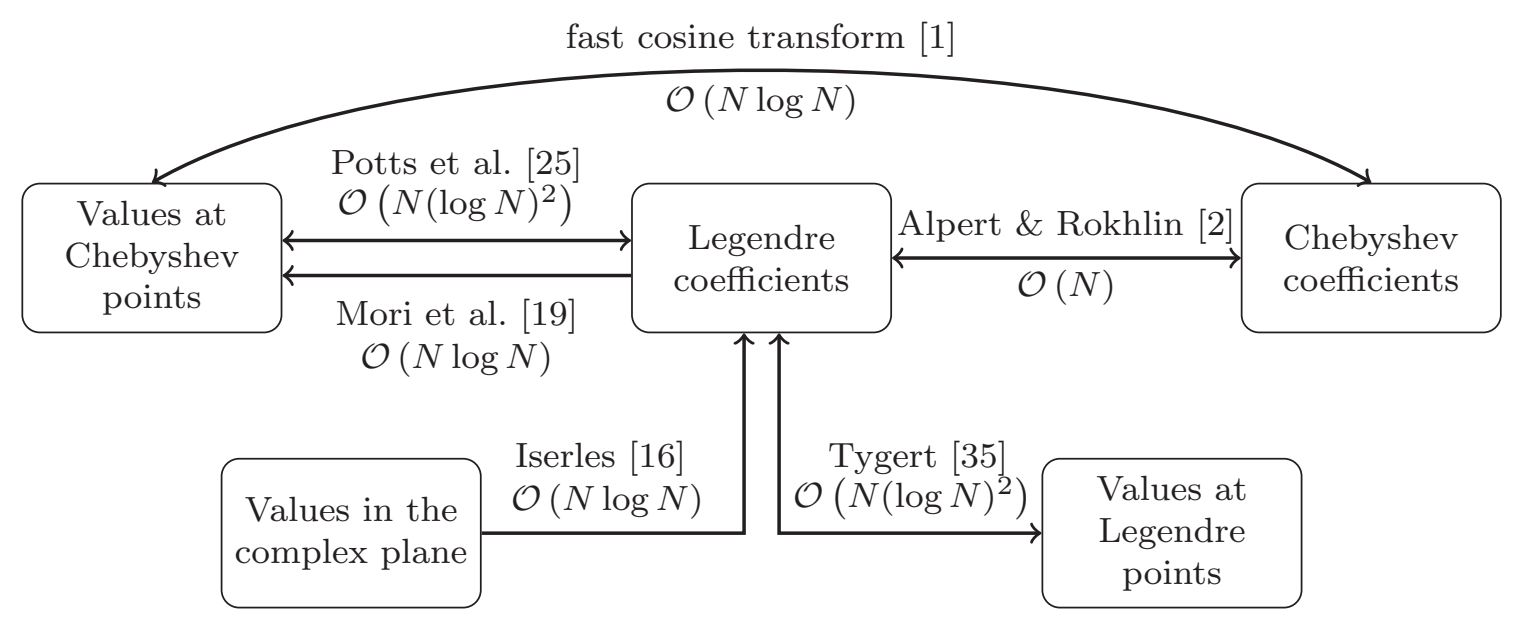

Fig. 2.1: Existing fast algorithms related to Chebyshev-Legendre transforms.

The algorithm we describe is based on Stieltjes' long-established asymptotic formula for Legendre polynomials [30], and can be seen as a numerically stable modification of the approach by Mori et al. [19]. As we shall explain, it can be interpreted as approximating the Legendre-Vandermonde-like matrix by a weighted linear combination of Chebyshev-Vandermonde-like matrices, whose action on vectors can be efficiently computed using the discrete cosine transform (DCT).

The outline of this paper is as follows: In the next section we discuss existing fast algorithms for the Chebyshev-Legendre transform and justify the need for a new approach. In Section 3 we discuss the forward transform. We begin by introducing the asymptotic formula of Stieltjes [30] and describing the numerically unstable algorithm of Mori et al. [19], before advocating a novel modification that leads to a fast and stable algorithm. In Section 4 we describe a similar new fast algorithm for the inverse transform, and in Section 5 we present numerical results for both algorithms. Finally, in Section 6 we discuss applications and future work for related fast transforms.

The code used for all the numerical results in this paper is publicly available from here [14]. It is also available in the Chebfun software system [34].

2. Existing methods. The problem of computing coefficients in a Legendre expansion has received considerable research attention since the 1970s [10,24]. These initial approaches required $\mathcal{O}\left(N^{2}\right)$ operations to compute the transform, and to the authors' knowledge the first algorithm for computing the coefficients of a Legendre expansion in less than $\mathcal{O}\left(N^{2}\right)$ operations is due to Orszag [23] in 1986. Later, in 1991, Alpert and Rokhlin [2] described an algorithm based on multipole-like ideas, requiring just $\mathcal{O}(N)$ operations. Since then many other fast algorithms have been proposed $[7,19,25,35]$. Figure 2.1 summarises the main algorithms, which are briefly described below.

2.1. Approaches using asymptotic expansions. Orzsag [23], in 1986, described a fast algorithm for eigenfunction transforms that can be used for the computation of Legendre coefficients. The algorithm is based on a first-order WKB expansion of Legendre polynomials, but it is not considered useful in practice as the expansion converges too slowly. The algorithm we present for computing the forward transform is similar to Orszag's approach, but improved in two crucial ways: (1) We use a different asymptotic formula for $P_{n}(x)$ due to Stieltjes that converges more rapidly [30,32]; and (2) We use an accompanying explicit error formula to derive the complexity and determine certain algorithmic constants of the transform. 
We are not the first to use Stieltjes' asymptotic formula for computing the fast transform as it was employed by Mori, Suda, and Sugihara [19] in 1999 to derive an algorithm requiring $\mathcal{O}(N \log N)$ operations. The algorithm described in [19] is fast and accurate for small $N$, but as $N$ increases it becomes numerically unstable in floating point arithmetic. Suda, Mori, and Sugihara were aware of the numerical instability in their algorithm and in 2002 began preparing a manuscript to fix the numerical issues. However, that work was not finished and they no longer intend to publish ${ }^{1}$. Furthermore, even with the unpublished modification (as noted in the manuscript), their algorithm is still unstable for large $N$. In this paper we present a further modification that is numerically stable for all $N$. In particular, in Section 3, we adapt the algorithm in [19] to derive a stable transform requiring $\mathcal{O}\left(N(\log N)^{2} / \log \log N\right)$ operations that can transform between one million Legendre and Chebyshev coefficients, or more.

2.2. The fast multipole method. The fast multipole-like approach described by Alpert and Rohklin [2] transforms between Legendre and Chebyshev coefficients in $\mathcal{O}(N)$ operations. The cost of the algorithm depends on the working precision, and for double precision arithmetic they observe that after the initialization phase it is about 5.5 times the cost of a single fast Fourier transform (FFT) of the same length [2]. Although this approach is often considered state-of-the-art, the algorithm is not widely used in practice as the initialization phase can be expensive and the hierarchical data structures required make it difficult to implement efficiently. The algorithms for the forward and inverse transforms presented in this paper do not require an initialization phase, and are sufficiently simple that they can be efficiently implemented in about 100 lines of MATLAB code (see Section 6).

2.3. Divide-and-conquer approaches. Potts, Steidl, and Tasche described in 1998 a fast algorithm that transforms between function values at Chebyshev points and Legendre coefficients [25]. The algorithm uses a divide-and-conquer approach and hierarchical data structures to apply the matrix-vector product involving the Legendre-Vandermonde-like matrix

$$
\mathbf{P}_{\mathbf{N}}\left(\underline{x}_{N}^{c h e b}\right)=\left[P_{0}\left(\underline{x}_{N}^{c h e b}\right)|\cdots| P_{N-1}\left(\underline{x}_{N}^{c h e b}\right)\right]
$$

in $\mathcal{O}\left(N(\log N)^{2}\right)$ operations, where $P_{0}, \ldots, P_{N-1}$ are the first $N$ Legendre polynomials and $\underline{x}_{N}^{c h e b}$ denotes the vector of $N$ Chebyshev points in decreasing order.

Tygert [35], in 2010, describes a similar algorithm, noting that the LegendreVandermonde-like matrix can be decomposed as $\mathbf{P}_{\mathbf{N}}\left(\underline{x}_{N}^{l e g}\right)=D_{\underline{w}} U D_{\underline{s}}$, where $\underline{x}_{N}^{\text {leg }}$ is the vector of $N$ Gauss-Legendre points, $D_{\underline{w}}$ is the diagonal matrix of GaussLegendre quadrature weights, $D_{\underline{s}}$ is the diagonal matrix of orthonormalization factors for Legendre polynomials, and $\bar{U}$ is an orthogonal matrix. Tygert then uses the fact that the orthogonal matrix $U$ can be applied in $\mathcal{O}(N \log N)$ operations since the columns are the eigenvectors of a symmetric tridiagonal matrix [12]. The approach proposed by Tygert is more general than just a fast Legendre transform and he notes that specialized algorithms are likely to be more efficient.

2.4. Function dependent approaches. In 2011, Iserles [16] described an algorithm to compute the fast Legendre coefficients from sampling a function at points lying on a certain Bernstein ellipse in the complex plane. The algorithm requires

\footnotetext{
${ }^{1}$ We are very grateful for private communication with Professor Reiji Suda from the University of Tokyo in June 2013.
} 
$\mathcal{O}(N \log N)$ operations and is much simpler to implement than other approaches mentioned thus far. However, the size of the Bernstein ellipse required depends on the region of analyticity of $f$, making the algorithm difficult to use in a black box manner. Furthermore, it seems that in practice this algorithm suffers from numerical instability for large $N(N \geq 512)$, and quadratic precision is required in the computations to get even double or single precision accuracy in the results.

More recently, De Micheli and Viano in [7] describe a fast algorithm based on integral transforms, which also depends on the smoothness of the prescribed function. The algorithm we derive does not depend on the smoothness of the function and is applicable to any vector of real or complex coefficients.

3. The forward transform: Legendre to Chebyshev. For notational convenience we express equations (1.1) and (1.2) in the form

$$
p_{N}(x)=\mathbf{T}_{\mathbf{N}}(x) \underline{c}_{N}^{c h e b}=\mathbf{P}_{\mathbf{N}}(x) \underline{c}_{N}^{l e g},
$$

where $x$ is an independent variable and

$$
\mathbf{T}_{\mathbf{N}}(x)=\left[T_{0}(x)|\ldots| T_{N}(x)\right], \quad \mathbf{P}_{\mathbf{N}}(x)=\left[P_{0}(x)|\ldots| P_{N}(x)\right],
$$

are Chebyshev and Legendre quasimatrices ${ }^{2}$, i.e., the $\infty \times(N+1)$ matrices that have in their $n$th column the degree $n-1$ Chebyshev and Legendre polynomial, respectively. If $-1 \leq x_{N}<\cdots<x_{0} \leq 1$ are $N+1$ distinct points in $[-1,1]$, indexed in reverse order to simplify later notation, and $\underline{x}_{N}=\left\{x_{j}\right\}_{0 \leq j \leq N}$, then

$$
p_{N}\left(\underline{x}_{N}\right)=\mathbf{T}_{\mathbf{N}}\left(\underline{x}_{N}\right) \underline{c}_{N}^{c h e b}=\mathbf{P}_{\mathbf{N}}\left(\underline{x}_{N}\right) \underline{c}_{N}^{l e g} .
$$

For brevity, we refer to the Vandermonde-like matrices $\mathbf{T}_{\mathbf{N}}\left(\underline{x}_{N}\right)$ and $\mathbf{P}_{\mathbf{N}}\left(\underline{x}_{N}\right)$ as the Chebyshev-Vandermonde and Legendre-Vandermonde matrices, respectively. Now, since polynomial interpolants at distinct points are unique, $\mathbf{T}_{\mathbf{N}}\left(\underline{x}_{N}\right)$ and $\mathbf{P}_{\mathbf{N}}\left(\underline{x}_{N}\right)$ are invertible, and we may write

$$
\underline{c}_{N}^{c h e b}=\mathbf{T}_{\mathbf{N}}\left(\underline{x}_{N}\right)^{-1} \mathbf{P}_{\mathbf{N}}\left(\underline{x}_{N}\right) \underline{c}_{N}^{l e g} .
$$

For a general vector of distinct points $\underline{x}_{N}$, a naive algorithm requires $\mathcal{O}\left(N^{2}\right)$ operations for a matrix-vector product with $\mathbf{P}_{\mathbf{N}}\left(\underline{x}_{N}\right)$, and $\mathcal{O}\left(N^{3}\right)$ operations to apply $\mathbf{T}_{\mathbf{N}}\left(\underline{x}_{N}\right)^{-1}$ to a vector. However, if $\underline{x}_{N}=\underline{x}_{N}^{c h e b}$, i.e., the vector of $N+1$ Chebyshev points (of the second kind),

$$
x_{k}^{\text {cheb }}=\cos (k \pi / N), \quad k=0, \ldots, N,
$$

then $\mathbf{T}_{\mathbf{N}}\left(\underline{x}_{N}^{c h e b}\right)$ is the matrix representing a $\mathrm{DCT}^{3}$, and can be applied and inverted in $\mathcal{O}(N \log N)$ operations $[1,11]$. Applying $\mathbf{P}_{\mathbf{N}}\left(\underline{x}_{N}^{c h e b}\right)$ to a vector in fewer than $\mathcal{O}\left(N^{2}\right)$ operations is less straight-forward, but in the following section we describe how this can be achieved by employing a well-known asymptotic formula.

As an aside, we note that if $\mathbf{T}_{\mathbf{N}}\left(\underline{x}_{N}^{\text {cheb }}\right)^{-1}$ is not applied, then $\mathbf{P}_{\mathbf{N}}\left(\underline{x}_{N}^{\text {cheb }}\right) \underline{c}_{N}^{\text {leg }}=$ $p_{N}\left(\underline{x}_{N}^{c h e b}\right)$ is simply $p_{N}(x)$ evaluated on the Chebyshev grid $\underline{x}_{N}^{c h e b}$, which is useful for the fast evaluation of a Legendre expansion and spectral collocation methods [6].

\footnotetext{
${ }^{2}$ The term quasimatrix was coined by Stewart in [29] to describe 'matrices' with columns consisting of functions.

${ }^{3}$ In this paper we use the acronym DCT to refer to the discrete cosine transform of type I (DCTI, [37]) with the first and last columns of the DCT-I matrix scaled, so that it equals the matrix $\mathbf{T}_{\mathbf{N}}\left(\underline{x}_{N}^{c h e b}\right)$. Moreover, the matrix $\mathbf{T}_{\mathbf{N}}\left(\underline{x}_{N}^{c h e b}\right)$ is symmetric, i.e., $\mathbf{T}_{\mathbf{N}}\left(\underline{x}_{N}^{c h e b}\right)^{T}=\mathbf{T}_{\mathbf{N}}\left(\underline{x}_{N}^{c h e b}\right)$.
} 
3.1. An asymptotic formula for Legendre polynomials. In 1890, Stieltjes [30] derived the following asymptotic formula for Legendre polynomials as $n \rightarrow \infty$ :

$$
P_{n}(\cos \theta)=C_{n} \sum_{m=0}^{M-1} h_{m, n} \frac{\cos \left(\left(m+n+\frac{1}{2}\right) \theta-\left(m+\frac{1}{2}\right) \frac{\pi}{2}\right)}{(2 \sin \theta)^{m+1 / 2}}+R_{M, n}(\theta),
$$

where $\theta=\cos x$ for $\theta \in(0, \pi)$, and

$$
\begin{gathered}
C_{n}=\frac{4}{\pi} \prod_{j=1}^{n} \frac{j}{j+1 / 2}=\sqrt{\frac{4}{\pi}} \frac{\Gamma(n+1)}{\Gamma(n+3 / 2)}, \\
h_{m, n}=\left\{\begin{array}{cc}
1, & m=0, \\
\prod_{j=1}^{m} \frac{(j-1 / 2)^{2}}{j(n+j+1 / 2)}, & m>0 .
\end{array}\right.
\end{gathered}
$$

Szegö, in his classic book on orthogonal polynomials [32], showed that the error term can be bounded by

$$
\left|R_{M, n}(\theta)\right| \leq C_{n} h_{M, n} \frac{2}{(2 \sin \theta)^{M+\frac{1}{2}}} .
$$

This upper bound is sharp and a good approximate lower bound is half the upper bound, since $\left|R_{M, n}(\theta)\right|$ is less than twice the first neglected term in (3.3) [32]. The bound on $R_{M, n}(\theta)$ shows that (3.3) converges to $P_{n}(\cos \theta)$ as $M \rightarrow \infty$ for $\theta \in(\pi / 6,5 \pi / 6)$, i.e., for $\theta$ such that $|2 \sin \theta|<1$. However, as suggested by Szegö in [32] and demonstrated in [5,15], for finite values of $M$ this asymptotic formula can still be an excellent approximation for $\theta \notin(\pi / 6,5 \pi / 6)$. In practice, if $n$ is sufficiently large, (3.3) can be used to approximate $P_{n}(\cos \theta)$ to double precision for almost all $\theta \in(0, \pi)$. The exact region in the $(n, \theta)$-plane in which $M$ terms of (3.3) approximates $P_{n}(\cos \theta)$ to a prescribed tolerance can be determined from (3.6), as we derive later in (3.14).

To make (3.3) amenable to evaluation using the DCT, we first note that $(m+n+$ $\left.\frac{1}{2}\right) \theta-\left(m+\frac{1}{2}\right)=n \theta-\left(m+\frac{1}{2}\right)\left(\frac{\pi}{2}-\theta\right)$ and rewrite the trigonometric term in (3.3) as

$$
\cos \left(\left(m+n+\frac{1}{2}\right) \theta-\left(m+\frac{1}{2}\right) \frac{\pi}{2}\right)=\cos \left(n \theta-\left(m+\frac{1}{2}\right)\left(\frac{\pi}{2}-\theta\right)\right) .
$$

Applying a standard trigonometric identity to $\cos (A-B)$ we find

$$
\begin{aligned}
\cos \left(n \theta-\left(m+\frac{1}{2}\right)\left(\frac{\pi}{2}-\theta\right)\right)=\sin (n \theta) & \sin \left(\left(m+\frac{1}{2}\right)\left(\frac{\pi}{2}-\theta\right)\right) \\
& +\cos (n \theta) \cos \left(\left(m+\frac{1}{2}\right)\left(\frac{\pi}{2}-\theta\right)\right) .
\end{aligned}
$$

Noting that $T_{n}(\cos \theta)=\cos (n \theta)$ and $U_{n-1}(\cos \theta)=\sin (n \theta) / \sin \theta$ are Chebyshev polynomials of the first and second kind, respectively, we have

$$
\begin{aligned}
\cos \left(n \theta-\left(m+\frac{1}{2}\right)\left(\frac{\pi}{2}-\theta\right)\right)=U_{n-1}(\cos \theta) & \sin \left(\left(m+\frac{1}{2}\right)\left(\frac{\pi}{2}-\theta\right)\right) \sin \theta \\
& +T_{n}(\cos \theta) \cos \left(\left(m+\frac{1}{2}\right)\left(\frac{\pi}{2}-\theta\right)\right) .
\end{aligned}
$$

Finally, substituting (3.7) back into (3.3), we find the asymptotic formula (3.3) can be expressed as a weighted linear combination of Chebyshev polynomials

$$
P_{n}(\cos \theta)=C_{n} \sum_{m=0}^{M-1} h_{m, n}\left(u_{m}(\theta) U_{n-1}(\cos \theta)+v_{m}(\theta) T_{n}(\cos \theta)\right)+R_{M, n}(\theta),
$$


where

$$
u_{m}(\theta)=\frac{\sin \left(\left(m+\frac{1}{2}\right)\left(\frac{\pi}{2}-\theta\right)\right) \sin \theta}{(2 \sin \theta)^{m+1 / 2}}, \quad v_{m}(\theta)=\frac{\cos \left(\left(m+\frac{1}{2}\right)\left(\frac{\pi}{2}-\theta\right)\right)}{(2 \sin \theta)^{m+1 / 2}} .
$$

Now, since $T_{n}(\cos \theta)$ and $U_{n-1}(\cos \theta)$ are the only terms in (3.8) that depend on both $n$ and $\theta$, the quasimatrix $\mathbf{P}_{\mathbf{N}}(x)$ from $(3.1)$ can be expressed in the following compact form:

$$
\mathbf{P}_{\mathbf{N}}(\cos \theta)=\sum_{m=0}^{M-1}\left(D_{u_{m}(\theta)}\left[0 \mid \mathbf{U}_{\mathbf{N}-\mathbf{1}}(\cos \theta)\right]+D_{v_{m}(\theta)} \mathbf{T}_{\mathbf{N}}(\cos \theta)\right) D_{\underline{C h}_{m}}+\mathbf{R}_{M}(\theta)
$$

where $x=\cos \theta, D_{u_{m}}(\theta)$ and $D_{v_{m}}(\theta)$ are the diagonal operators with $u_{m}(\theta)$ and $v_{m}(\theta)$ from (3.9) on the diagonal, $D_{\underline{C h}_{m}}$ is the diagonal matrix of the pointwise product of (3.4) and (3.5) for $n=0, \ldots, N$, and $\mathbf{R}_{M}(\theta)=\left[R_{M, 0}(\theta)|\ldots| R_{M, N}(\theta)\right]$.

Substituting $x=\underline{x}_{N}^{c h e b}=\cos \left(\underline{\theta}_{N}^{\text {cheb }}\right)$ means that $\mathbf{U}_{\mathbf{N}-\mathbf{1}}\left(\underline{x}_{N}^{\text {cheb }}\right)$ and $\mathbf{T}_{\mathbf{N}}\left(\underline{x}_{N}^{\text {cheb }}\right)$ are essentially discrete cosine/sine transformation matrices, which can be applied to a vector in $\mathcal{O}(N \log N)$ operations using the DCT. Since $\mathbf{P}_{\mathbf{N}}\left(\underline{x}_{N}^{\text {cheb }}\right)$ is simply a diagonally-weighted linear combination of these matrices, the matrix-vector product $\mathbf{P}_{\mathbf{N}}\left(\underline{x}_{N}^{c h e b}\right) \underline{c}_{N}^{c h e b}$ in $(3.10)$ can be evaluated in $\mathcal{O}(M N \log N)$ operations using (3.10) with an error of $\mathbf{R}_{M}\left(\underline{\theta}_{N}^{\text {cheb }}\right) \underline{c}_{N}^{\text {cheb }}$.

3.2. Partitioning the Legendre-Vandermonde matrix for the forward transform. First, we take the unusual step of describing an unstable algorithm by Mori et al. [19] for the forward transform that we do not advocate. However, by describing this algorithm now we motivate and set the scene for its stable variant (see Section 3.3).

This unstable algorithm computes $\mathbf{P}_{\mathbf{N}}\left(\underline{x}_{N}^{c h e b}\right) c_{N}^{\text {leg }}$ by partitioning $\mathbf{P}_{\mathbf{N}}\left(\underline{x}_{N}^{c h e b}\right)$ into two matrices, denoted by $\mathbf{P}_{\mathbf{N}}^{\mathrm{REC}}\left(\underline{x}_{N}^{\text {cheb }}\right)$ and $\mathbf{P}_{\mathbf{N}}^{\mathrm{DCT}}\left(\underline{x}_{N}^{\text {cheb }}\right)$. Making use of discrete cosine transforms, the matrix $\mathbf{P}_{\mathbf{N}}^{\mathrm{DCT}}\left(\underline{x}_{N}^{\text {cheb }}\right)$ is applied to a vector via the asymptotic formula (3.10), and in this process an unacceptably large error for certain $(n, \theta)$ can be committed which must be corrected by a matrix $\mathbf{P}_{\mathbf{N}}^{\mathrm{COR}}\left(\underline{x}_{N}^{c h e b}\right)$. Effectively, this algorithm expresses $\mathbf{P}_{\mathbf{N}}\left(\underline{x}_{N}^{c h e b}\right)$ as a sum of three matrices,

$$
\mathbf{P}_{\mathbf{N}}\left(\underline{x}_{N}^{c h e b}\right)=\mathbf{P}_{\mathbf{N}}^{\mathrm{REC}}\left(\underline{x}_{N}^{c h e b}\right)+\mathbf{P}_{\mathbf{N}}^{\mathrm{DCT}}\left(\underline{x}_{N}^{c h e b}\right)+\mathbf{P}_{\mathbf{N}}^{\mathrm{COR}}\left(\underline{x}_{N}^{c h e b}\right),
$$

where the matrices are shown in Figure 3.1 (left). The matrix $\mathbf{P}_{\mathbf{N}}^{\mathrm{REC}}\left(\underline{x}_{N}^{c h e b}\right)$ contains all the columns and rows of $\mathbf{P}_{\mathbf{N}}\left(\underline{x}_{N}^{c h e b}\right)$ that do not intersect the error curve $\left|R_{M, n}(\theta)\right|=\epsilon$, that is,

$$
\mathbf{P}_{\mathbf{N}}^{\mathrm{REC}}\left(\underline{x}_{N}^{\text {cheb }}\right)_{i j}= \begin{cases}\mathbf{P}_{\mathbf{N}}\left(\underline{x}_{N}^{c h e b}\right)_{i j}, & 1 \leq \min (i, N-i+1) \leq j_{M}, \\ \mathbf{P}_{\mathbf{N}}\left(\underline{x}_{N}^{c h e b}\right)_{i j}, & 1 \leq j \leq n_{M}, \\ 0, & \text { otherwise }\end{cases}
$$

Here, $n_{M}$ is the number of Legendre polynomials, $P_{0}, \ldots, P_{n_{M}}$, that cannot be approximated using the asymptotic formula (3.3) to a precision $\epsilon$ at $\theta=\pi / 2(x=0)$. In other words, $\left|R_{M, n}(\pi / 2)\right|<\epsilon$ for all $n>n_{M}$. It can be shown, using (3.6) and its approximate sharpness, that for $n \gg M$,

$$
\left|R_{M, n}(\pi / 2)\right| \gtrsim C_{n} h_{n, M} \frac{1}{2^{M+1 / 2}}=\mathcal{O}\left(\frac{4 \Gamma(M+1 / 2)^{2}}{\pi^{3 / 2} \Gamma(M+1)} \frac{n^{-M-1 / 2}}{2^{M+\frac{1}{2}}}\right)
$$




\begin{tabular}{|c|c|c|c|c|c|c|c|c|c|c|c|c|c|}
\hline $\mathrm{M}$ & 3 & 4 & 5 & 6 & 7 & 8 & 9 & 10 & 11 & 12 & 13 & 14 & 15 \\
\hline$n_{M}$ & 16,072 & 2,053 & 583 & 252 & 139 & 90 & 65 & 50 & 41 & 35 & 30 & 27 & 25 \\
$j_{M}$ & 5,000 & 658 & 185 & 80 & 44 & 28 & 20 & 15 & 13 & 11 & 9 & 8 & 7 \\
\hline
\end{tabular}

Table 3.1: Algorithmic constants for $3 \leq M \leq 15$ and $\epsilon=2.2 \times 10^{-16}$ in the regime of $N \gg n_{M}$ and $n \gg M . P_{n_{M}+1}(x)$ is the lowest degree Legendre polynomial that is evaluated at $x=0$ to machine precision using $M$ terms in the asymptotic formula, and $j_{M}$ is such that $P_{N}\left(x_{i}\right)$ is evaluated to machine precision for any $j_{M} \leq i \leq N-j_{M}$.

where the last equality is the leading term in a series expansion of $R_{M, n}(\pi / 2)$ for $n \gg M$. Solving (3.12) we obtain,

$$
n_{M}=\left\lfloor\frac{1}{2}\left(\epsilon \frac{\pi^{3 / 2} \Gamma(M+1)}{4 \Gamma(M+1 / 2)^{2}}\right)^{\frac{-1}{M+\frac{1}{2}}}\right\rfloor,
$$

and Table 3.1 gives some values of $n_{M}$ for $3 \leq M \leq 15$. More generally, we can use (3.12) to derive the following error curve:

$$
\left|R_{M, n}(\theta)\right|=\epsilon \quad \Longrightarrow \quad n \approx n_{M} / \sin \theta .
$$

The curves clearly depend on $M$, and Figure 3.1 (right) depicts those for $M=$ $5,7,10,15$ and $N=1,000$. Similar figures appear in [19].

$j_{M}$ gives the number of values $P_{N}\left(x_{0}\right), \ldots, P_{N}\left(x_{j_{M}-1}\right)$ that cannot be approximated by the asymptotic formula (3.3) to a tolerance of $\epsilon$ using $M$ terms in the asymptotic formula. Thus, using (3.14), $j_{M}$ is the number of points in the interval

$$
0 \leq \theta \leq \sin ^{-1}\left(\frac{n_{M}}{N}\right)
$$

Since $\cos ^{-1}\left(x_{0}\right), \ldots, \cos ^{-1}\left(x_{j_{M}}\right)$ are equally-spaced with spacing $\pi /(N+1)$ in the $\theta$-variable we have,

$$
j_{M}=\left\lfloor\frac{N+1}{\pi} \sin ^{-1}\left(\frac{n_{M}}{N}\right)\right\rfloor .
$$

Moreover, $\sin ^{-1}(x) \approx x$ for $|x| \ll 1$ and hence, for $N \gg n_{M}$ the parameter $j_{M}$ is essentially independent of $N$. Table 3.1 gives the values of $j_{M}$ for $3 \leq M \leq 15$.

Note that equations (3.13) and (3.15) for the algorithmic constants $n_{M}$ and $j_{M}$ assume that $n \gg M$ and $N \gg n_{M}$, respectively. In fact, the analysis here is not intended to be overly rigorous or technical, but just give an estimates of the error curve, $n_{M}$, and $j_{M}$. A more technical analysis can be performed taking more care when certain series expansions are employed, but this does not significantly change the practical properties of the algorithm.

We compute the resulting vector $\mathbf{P}_{\mathbf{N}}\left(\underline{x}_{N}^{c h e b}\right) c_{N}^{l e g}$ by applying the three matrices in (3.11) separately. The matrix-vector product $\mathbf{P}_{\mathbf{N}}^{\mathrm{REC}}\left(\underline{x}_{N}^{c h e b}\right) \underline{s}_{N}^{\text {leg }}$ can be computed in $\mathcal{O}(N)$ operations because the matrix $\mathbf{P}_{\mathbf{N}}^{\mathrm{REC}}\left(\underline{x}_{N}^{c h e b}\right)$ has fewer than $\left(2 j_{M}+n_{M}\right) N=$ $\mathcal{O}(N)$ nonzero entries. These entries cannot be computed via the asymptotic formula (3.3) because for these $(n, \theta)$ we have $\left|R_{M, n}(\theta)\right|>\epsilon$. Instead, we use the well-known three-term recurrence relation satisfied by Legendre polynomials [21]:

$$
P_{n+1}(x)=\left(2-\frac{1}{n+1}\right) x P_{n}(x)-\left(1-\frac{1}{n+1}\right) P_{n-1}(x), \quad n \geq 1 .
$$



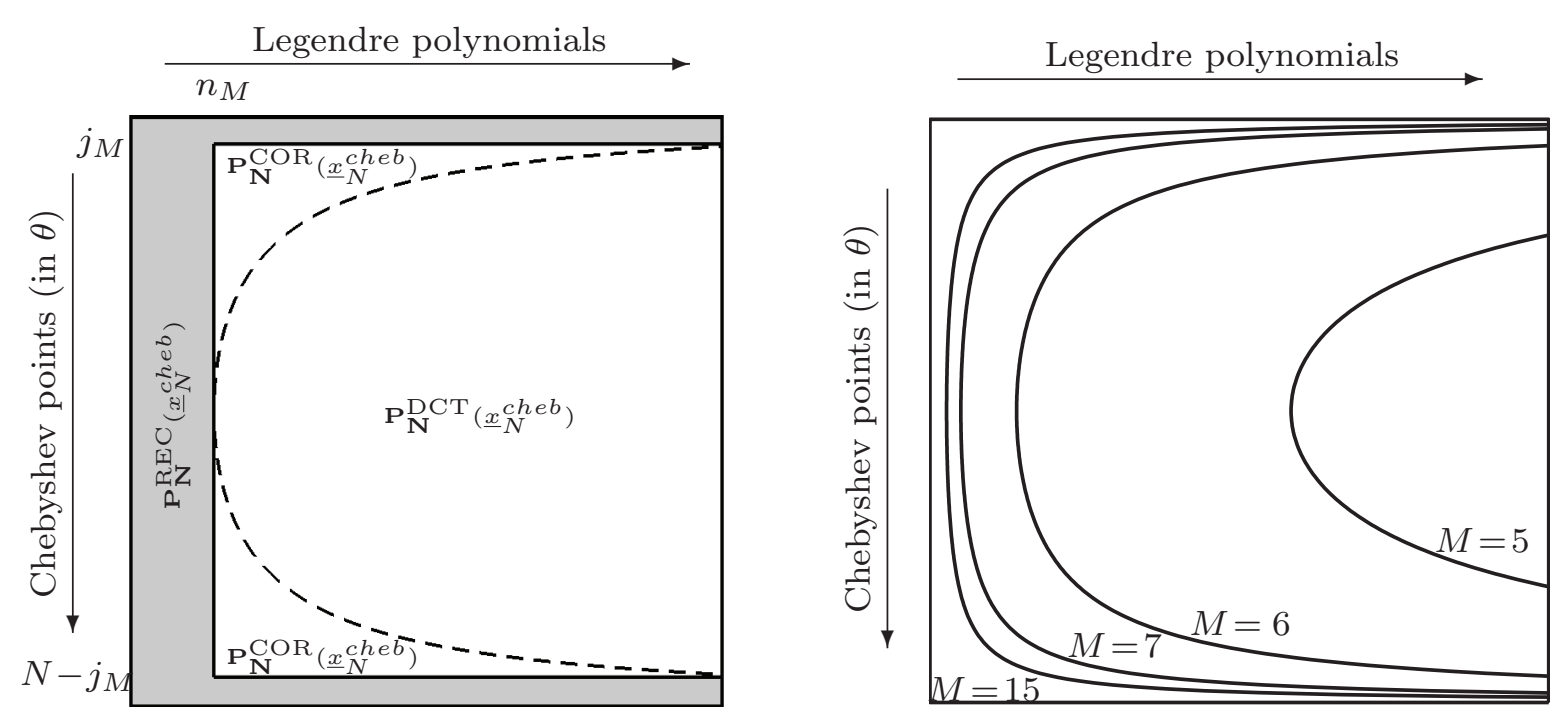

Fig. 3.1: Left: The partition of the matrix $\mathbf{P}_{\mathbf{N}}\left(\underline{x}_{N}^{c h e b}\right)$ employed in the unstable algorithm. The dashed line indicates the boundary of the region in which the asymptotic formula can be employed without correction, and the gray region indicates the nonzero entries of the matrix $\mathbf{P}_{\mathbf{N}}^{\mathrm{REC}}\left(\underline{x}_{N}^{\text {cheb }}\right)$. Right: The error curves $\left|R_{M, n}(\theta)\right|=\epsilon$ for $M=5,6,7,15$ and $N=1,000$.

In this way, $\mathbf{P}_{\mathbf{N}}^{\mathrm{REC}}\left(\underline{x}_{N}^{c h e b}\right) \underline{c}_{N}^{\text {leg }}$ is computed without explicitly forming $\mathbf{P}_{\mathbf{N}}^{\mathrm{REC}}\left(\underline{x}_{N}^{c h e b}\right)$.

For the matrix-vector product $\mathbf{P}_{\mathbf{N}}^{\mathrm{DCT}}\left(\underline{x}_{N}^{c h e b}\right) \underline{c}_{N}^{\text {leg }}$ we write $\mathbf{P}_{\mathbf{N}}^{\mathrm{DCT}}\left(\underline{x}_{N}^{c h e b}\right)$ as the weighted sum of Chebyshev-Vandermonde matrices given in (3.10). However, since the first $n_{M}$ columns and first and last $j_{M}$ rows of $\mathbf{P}_{\mathbf{N}}^{\mathrm{DCT}}\left(\underline{x}_{N}^{c h e b}\right)$ are zero we must restrict the DCTs when applying the matrices $\mathbf{U}_{\mathbf{N}-\mathbf{1}}\left(\underline{x}_{N}^{\text {cheb }}\right)$ and $\mathbf{T}_{\mathbf{N}}\left(\underline{x}_{N}^{\text {cheb }}\right)$. One can think of this as pre- and post-multiplying the Chebyshev-Vandermonde matrices by identity matrices with the first and last $j_{M}$ and first $n_{M}$ entries on the diagonal zeroed, respectively. As before, each multiplication by the Chebyshev-Vandermonde matrices can be computed in $O(N \log N)$ operations using the DCT.

Unfortunately, in computing $\mathbf{P}_{\mathbf{N}}^{\mathrm{DCT}}\left(\underline{x}_{N}^{\text {cheb }}\right) \underline{\underline{w}}_{N}^{\text {leg }}$ we have employed the asymptotic formula in a region where $\left|R_{M, n}(\theta)\right|>\epsilon$, and we must correct for this. To do so, we construct a correction matrix $\mathbf{P}_{\mathbf{N}}^{\mathrm{COR}}\left(\underline{x}_{N}^{\text {cheb }}\right)$, see Figure 3.1 (left), with each nonzero entry equal to the true value of a Legendre polynomial minus the erroneous evaluation via the asymptotic formula (3.3). Thus, to compute each entry of $\mathbf{P}_{\mathbf{N}}^{\mathrm{COR}}\left(\underline{x}_{N}^{\text {cheb }}\right)$ we evaluate the Legendre polynomial using the three-term recurrence (3.16) and subtract the value obtained from the asymptotic formula in the form (3.3). Fortunately, as can be derived by the analysis in [19], the matrix $\mathbf{P}_{\mathbf{N}}^{\mathrm{COR}}\left(\underline{x}_{N}^{\text {cheb }}\right)$ contains $\mathcal{O}(N \log N)$ nonzero entries and thus, the correction vector $\mathbf{P}_{\mathbf{N}}^{\mathrm{COR}}\left(\underline{x}_{N}^{c h e b}\right) \underline{c}_{N}^{\text {leg }}$ can be computed in $\mathcal{O}(N \log N)$ operations. Since each of the matrices on the right-hand side of (3.2) can be applied in $\mathcal{O}(N \log N)$ operations, so can $\mathbf{P}_{N}\left(\underline{x}_{N}^{\text {cheb }}\right)$, and hence the entire forward transformation.

The major problem with this algorithm, as described, is that for any $M$ the transform becomes numerically unstable for sufficiently large $N$. The reason for this is cancellation error in floating point arithmetic. For large $N$ the asymptotic formula can erroneously evaluate to arbitrarily large values outside the dashed line in Figure 3.1 (left), which means the entries in $\mathbf{P}_{\mathbf{N}}^{\mathrm{COR}}\left(\underline{x}_{N}^{\text {cheb }}\right)$ lose all precision. This effect appears in practice, and in Figure 3.2 (left) we show the absolute error in the computed Chebyshev coefficients for various values of $M$ between 5 and 20 with 1,000 $\leq N \leq$ 10,000 . It is the cancellation error in computing the entries of $\mathbf{P}_{\mathbf{N}}^{\mathrm{COR}}\left(\underline{x}_{N}^{\text {cheb }}\right)$ that makes the algorithm numerically unstable and therefore, for large $N$, it is not as useful as one might hope for computing the forward transform. 

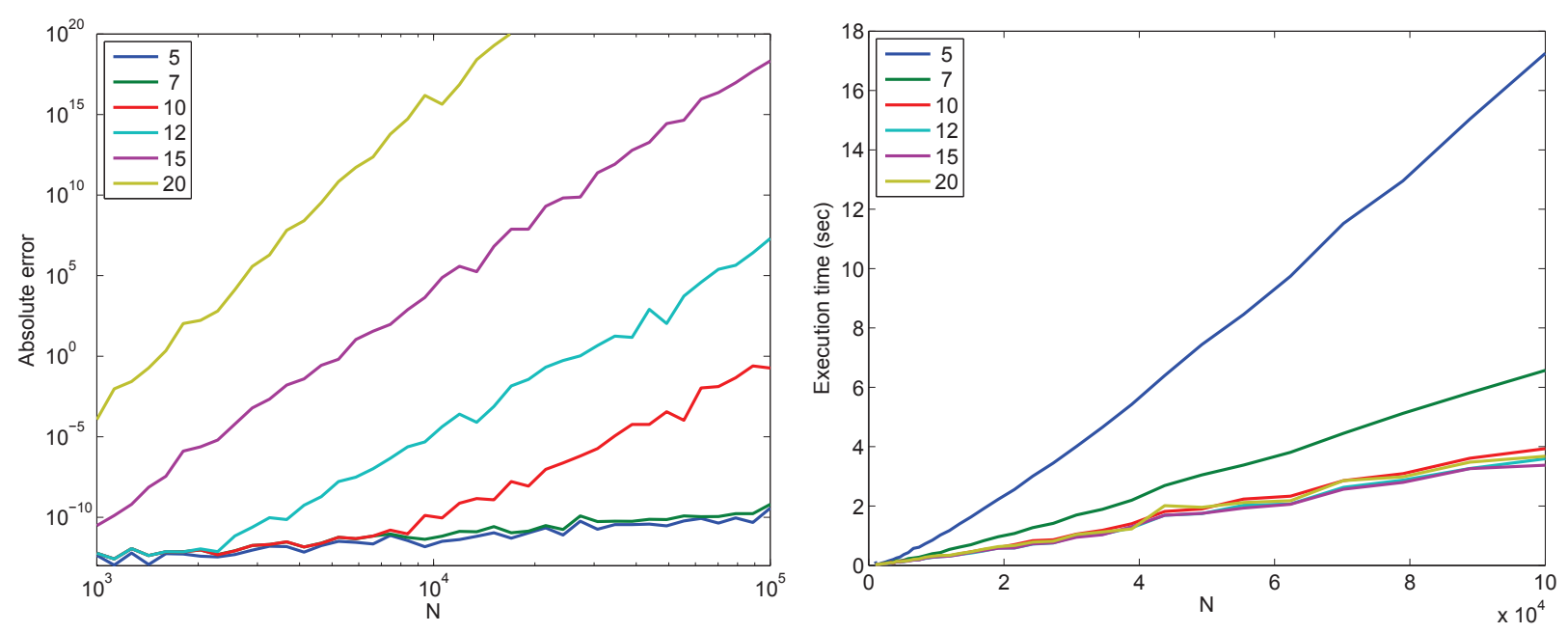

Fig. 3.2: Left: The maximum error in the computed coefficients $\underline{c}_{N}^{c h e b}$ for various $M$. For every $M \geq 1$ there is an integer $N_{\max }$ such that the algorithm is numerically unstable for $n>N_{\max }$. This situation can be remedied using the algorithm described in Section 3.3. Right: The execution times for the same values of $M$ and $10^{3} \leq N \leq 10^{5}$. By selecting a small value of $M$ one can ensure that the instability occurs only at a large $N$, but then the algorithm is much less efficient.

3.3. Block partitioning for numerical stability. Now we describe the algorithm that we do advocate for the forward transform based on a different partitioning of the Legendre-Vandermonde matrix $\mathbf{P}_{\mathbf{N}}\left(\underline{x}_{N}^{c h e b}\right)$. The algorithm is numerically stable and computes the vector $\mathbf{T}_{\mathbf{N}}\left(\underline{x}_{N}^{c h e b}\right)^{-1} \mathbf{P}_{\mathbf{N}}\left(\underline{x}_{N}^{c h e b}\right) \underline{c}_{N}^{l e g}$ in $\mathcal{O}\left(N(\log N)^{2} / \log \log N\right)$ operations.

We partition the matrix $\mathbf{P}_{\mathbf{N}}\left(\underline{x}_{N}^{c h e b}\right)$ into $K+1$ submatrices, where $K$ grows like $\mathcal{O}(\log N / \log \log N)$, in such a way that each submatrix can be applied to the vector $c_{N}^{l e g}$ in at most $\mathcal{O}(N \log N)$ operations. In particular, we partition $\mathbf{P}_{\mathbf{N}}\left(\underline{x}_{N}^{\text {cheb }}\right)$ as

$$
\mathbf{P}_{\mathbf{N}}\left(\underline{x}_{N}^{c h e b}\right)=\mathbf{P}_{\mathbf{N}}^{\mathrm{REC}}\left(\underline{x}_{N}^{c h e b}\right)+\sum_{k=1}^{K} \mathbf{P}_{\mathbf{N}}^{(k)}\left(\underline{x}_{N}^{c h e b}\right)
$$

where, for $k=1, \ldots, K$, we have

$$
\mathbf{P}_{\mathbf{N}}^{(k)}\left(\underline{x}_{N}^{c h e b}\right)= \begin{cases}\mathbf{P}_{\mathbf{N}}\left(\underline{x}_{N}^{c h e b}\right)_{i j}, & i_{k} \leq i \leq N-i_{k}, \quad \alpha^{k} N \leq j \leq \alpha^{k-1} N \\ 0, & \text { otherwise },\end{cases}
$$

$\alpha=\mathcal{O}(1 / \log N)$, and

$$
i_{k}=\left\lfloor\frac{N+1}{\pi} \sin ^{-1}\left(\frac{n_{M}}{\alpha^{k} N}\right)\right\rfloor
$$

The value of $i_{k}$ is the row index such that the submatrix $\mathbf{P}_{\mathbf{N}}{ }^{(k)}\left(\underline{x}_{N}^{c h e b}\right)$ nearly touches the error curve (3.14), and hence has a similar form as $j_{M}$ in (3.15) with $N$ replaced by $\alpha^{k} N$. Note that there is no need for a correction matrix $\mathbf{P}_{\mathbf{N}}^{\mathrm{COR}}\left(\underline{x}_{N}^{\text {cheb }}\right)$ in this algorithm, and that the matrix $\mathbf{P}_{\mathbf{N}}^{\mathrm{REC}}\left(\underline{x}_{N}^{\text {cheb }}\right)$ is defined slightly differently than in Section 3.2. The partitioning is shown in Figure 3.3 for $K=3$, and we remark that since $K$ grows relatively slowly with $N$, we require $N \geq 100,000$ for our algorithm to use $K \geq 3$ and $N \geq 10^{6}$ for $K \geq 4$.

This partitioning separates the matrix $\mathbf{P}_{\mathbf{N}}\left(\underline{x}_{N}^{c h e b}\right)$ into submatrices $\mathbf{P}_{\mathbf{N}}^{(k)}\left(\underline{x}_{N}^{c h e b}\right)$ whose nonzero entries can be computed by using the asymptotic formula without 


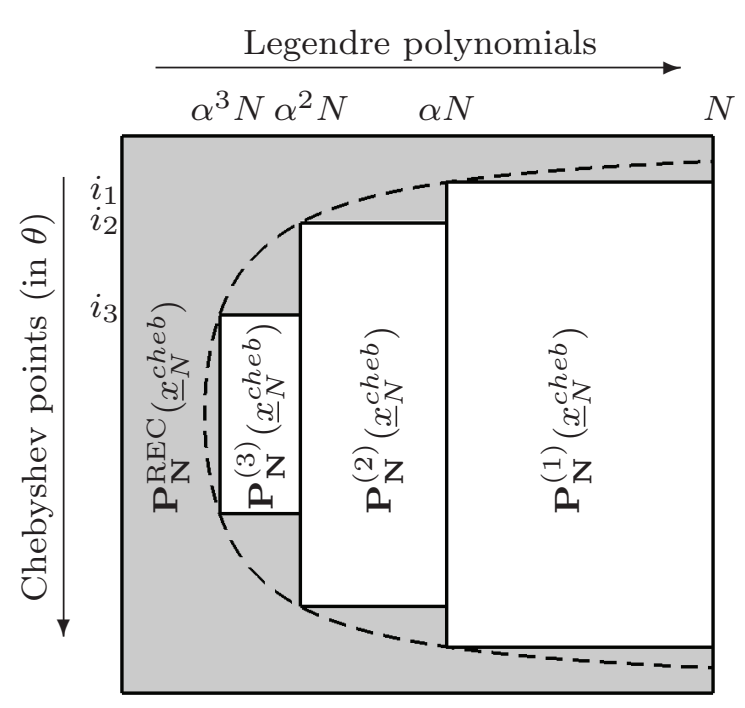

Fig. 3.3: Partitioning of the matrix $\mathbf{P}_{\mathbf{N}}\left(\underline{x}_{N}^{c h e b}\right)$ employed to compute the forward transform. The dashed line indicates the boundary of the region in which the asymptotic formula can be used without correction, and the gray region indicates the nonzero entries of the matrix $\mathbf{P}_{\mathbf{N}}^{\mathrm{REC}}\left(\underline{x}_{N}^{c h e b}\right)$. The diagram is not drawn to scale, and in practice the submatrix $\mathbf{P}_{\mathbf{N}}^{\mathrm{REC}}\left(\underline{x}_{N}^{c h e b}\right)$ occupies just a tiny proportion of $P_{N}\left(\underline{x}_{N}^{c h e b}\right)$.

correction, and in such a way that the cost of computing $\mathbf{P}_{\mathbf{N}}\left(\underline{x}_{N}^{c h e b}\right) \underline{c}_{N}^{\text {leg }}$ is minimal. The minimal cost is achieved, up to a constant, by balancing the cost of computing $\mathbf{P}_{\mathbf{N}}^{\mathrm{REC}}\left(\underline{x}_{N}^{c h e b}\right) \underline{c}_{N}^{l e g}$ with the cost of the $K$ matrix-vector products $\mathbf{P}_{\mathbf{N}}^{(k)}\left(\underline{x}_{N}^{c h e b}\right) \underline{c}_{N}^{l e g}$ for $k=1, \ldots, K$.

As we show later, the matrix $\mathbf{P}_{\mathbf{N}}^{\mathrm{REC}}\left(\underline{x}_{N}^{\text {cheb }}\right)$ contains $\mathcal{O}(K N / \alpha)$ nonzero entries and hence, can be applied to a vector in $\mathcal{O}(K N / \alpha)$ operations. In a similar fashion to the algorithm described in Section 3.2, we compute the nonzero entries of $\mathbf{P}_{\mathbf{N}}^{\mathrm{REC}}\left(\underline{x}_{N}^{\text {cheb }}\right)$ by using the three-term recurrence relation for Legendre polynomials (3.16).

The other matrices $\mathbf{P}_{\mathbf{N}}^{(k)}\left(\underline{x}_{N}^{c h e b}\right)$ for $k=1, \ldots, K$ are applied to a vector in $\mathcal{O}(N \log N)$ operations by employing the asymptotic formula (3.10) evaluated via the DCT. Notice that the nonzero entries of $\mathbf{P}_{\mathbf{N}}^{(k)}\left(\underline{x}_{N}^{c h e b}\right)$ form a rectangular submatrix of $\mathbf{P}_{\mathbf{N}}\left(\underline{x}_{N}^{c h e b}\right)$ and therefore, the matrix-vector product $\mathbf{P}_{\mathbf{N}}^{(k)}\left(\underline{x}_{N}^{c h e b}\right) \underline{c}_{N}^{\text {leg }}$ can be computed by restricting the DCTs when applying the matrices $\mathbf{U}_{\mathbf{N}-\mathbf{1}}\left(\underline{x}_{N}^{c h e b}\right)$ and $\mathbf{T}_{\mathbf{N}}\left(\underline{x}_{N}^{c h e b}\right)$. Again, one can think of this as pre- and post-multiplying the ChebyshevVandermonde matrices by identity matrices with certain entries on the diagonal zeroed out.

We now tidy up some unfinished business and detail how to partition the matrix $\mathbf{P}_{\mathbf{N}}\left(\underline{x}_{N}^{c h e b}\right)$ in (3.17) and analyze the complexity of the resulting algorithm. First, the number of nonzero entries in $\mathbf{P}_{\mathbf{N}}^{\mathrm{REC}}\left(\underline{x}_{N}^{c h e b}\right)$ can be calculated by artificially cutting it up into rectangular regions, and to leading order has

$$
\begin{aligned}
2\left(\sum_{k=1}^{K-1} \alpha^{k} N\left(i_{k+1}-i_{k}\right)\right) & \approx 2\left(\sum_{k=1}^{K-1} \alpha^{k} N \frac{N}{\pi}\left(\sin ^{-1}\left(\frac{n_{M}}{\alpha^{k+1} N}\right)-\sin ^{-1}\left(\frac{n_{M}}{\alpha^{k} N}\right)\right)\right) \\
& \approx \frac{2}{\pi} K N\left(\frac{1}{\alpha}-1\right)
\end{aligned}
$$

nonzero entries, where the last approximation uses $\sin ^{-1}(x) \approx x$, for $|x| \ll 1$. Therefore, the leading order cost of computing $\mathbf{P}_{\mathbf{N}}^{(k)}\left(\underline{x}_{N}^{c h e b}\right) \underline{c}_{N}^{\text {leg }}$ is $\mathcal{O}(K N / \alpha)$ operations, and we want to balance this with the $\mathcal{O}(K N \log N)$ cost of computing $\mathbf{P}_{\mathbf{N}}^{\mathrm{REC}}\left(\underline{x}_{N}^{\text {cheb }}\right) \underline{c}_{N}^{\text {leg }}$ for $k=1, \ldots, K$. To balance we should select $\alpha$ such that $K N / \alpha=K N \log N$, i.e., $\alpha=\mathcal{O}(1 / \log N)$. In practice, we have found that $\alpha=\left(1 / \log \left(N / n_{M}\right)\right)$ is a $\operatorname{good}$ 
choice for large $N$, and to avoid this becoming too close to 1 when $N$ is small we take

$$
\alpha=\min \left(1 / \log \left(N / n_{M}\right), 1 / 2\right) .
$$

Moreover, this discussion also determines $K$ since we need to partition the matrix $\mathbf{P}_{\mathbf{N}}\left(\underline{x}_{N}^{\text {cheb }}\right)$ into $K+1$ parts so that $\alpha^{K+1} N<n_{M}$ and therefore, we have

$$
K=\mathcal{O}(\log N / \log \log N)
$$

Putting this together, we have described an algorithm for the forward transform that requires $\mathcal{O}(K N \log N)$ operations, i.e., $\mathcal{O}\left(N(\log N)^{2} / \log \log N\right)$ operations. Furthermore, since the algorithm only employs the asymptotic formula for $(n, \theta)$ where $\left|R_{M, n}(\theta)\right|<\epsilon$, the transform is numerically stable. Additionally, the block partitioning means almost all computations can be vectorized, and since each of the $K+1$ matrix-vector multiplications as well as the DCTs in the asymptotic formula are independent, the algorithm is trivially parallelizable.

4. The inverse transform: Chebyshev to Legendre. The inverse transform converts a vector of Chebyshev coefficients, $\underline{c}_{N}^{c h e b}$, to a vector of Legendre coefficients, $\underline{c}_{N}^{l e g}$. Similarly to the forward transform, it can represented by a matrix-vector product involving Chebyshev- and Legendre-Vandermonde matrices:

$$
\underline{c}_{N}^{l e g}=\mathbf{P}_{\mathbf{N}}\left(\underline{x}_{N}^{c h e b}\right)^{-1} \mathbf{T}_{\mathbf{N}}\left(\underline{x}_{N}^{c h e b}\right) \underline{c}_{N}^{c h e b} .
$$

We begin with the integral definition for the Legendre coefficients, i.e.,

$$
c_{n}^{l e g}=\frac{1}{\left\|P_{n}\right\|_{2}^{2}} \int_{-1}^{1} p_{N}(x) P_{n}(x) d x, \quad 0 \leq k \leq N,
$$

where $P_{n}(x)$ is the degree $n$ Legendre polynomial, and $p_{N}(x)=\mathbf{T}_{\mathbf{N}}(x) \underline{c}_{N}^{c h e b}$ is the polynomial with Chebyshev coefficients $\underline{c}_{N}^{c h e b}$ as in (1.1). Since $p_{N}(x)$ is a polynomial of degree at most $N$, for any $0 \leq n \leq N$ the integrand, $p(x) P_{n}(x)$, is a polynomial of degree at most $2 N$, and the $(2 N+1)$-point Clenshaw-Curtis quadrature rule is exact for all the integrals in (4.2). Therefore, the Legendre coefficients satisfy the following discrete sums:

$$
c_{n}^{l e g}=\frac{1}{\left\|P_{n}\right\|_{2}^{2}} \sum_{j=0}^{2 N} w_{j} p_{N}\left(x_{j}\right) P_{n}\left(x_{j}\right), \quad 0 \leq n \leq N
$$

where $-1 \leq x_{2 N}<\cdots<x_{0} \leq 1$ and $w_{2 N}, \ldots, w_{0}$ are the Clenshaw-Curtis quadrature nodes and weights, respectively. Again, we have indexed the nodes in reverse order for easier notation later. Note that these Clenshaw-Curtis nodes are just the Chebyshev points from $(3.2)$ (with $N$ replaced by $2 N$ ) and hence, $\underline{x}_{2 N}^{c h e b}=\left(x_{0}, \ldots, x_{2 N}\right)^{T}$. Moreover, denote by $\underline{w}_{2 N}$ the vector of Clenshaw-Curtis weights, $\underline{w}_{2 N}=\left(w_{0}, \ldots, w_{2 N}\right)^{T}$, which can be computed in $O(N \log N)$ operations using the algorithm of Waldvogel [36], and $\underline{s}_{2 N}$, the orthonormalization vector for Legendre polynomials, $\underline{s}_{2 N}=$ $\left(\left\|P_{0}\right\|_{2}^{-2}, \ldots,\left\|P_{2 N}\right\|_{2}^{-2}\right)^{T}$. With this notation, (4.2) takes the following compact form:

$$
\begin{aligned}
\underline{c}_{N}^{l e g} & =\left[I_{N+1} \mid \mathbf{0}_{N}\right] D_{\underline{s}_{2 N}} \mathbf{P}_{\mathbf{2 N}}\left(\underline{x}_{2 N}^{\text {cheb }}\right)^{T} D_{\underline{w}_{2 N}} p_{N}\left(\underline{x}_{2 N}^{c h e b}\right) \\
& =\left[I_{N+1} \mid \mathbf{0}_{N}\right] D_{\underline{s}_{2 N}} \mathbf{P}_{\mathbf{2 N}}\left(\underline{x}_{2 N}^{c h e b}\right)^{T} D_{\underline{w}_{2 N}} \mathbf{T}_{\mathbf{2 N}}\left(\underline{x}_{2 N}^{c h e b}\right)\left[\begin{array}{c}
I_{N+1} \\
\mathbf{0}_{N}
\end{array}\right] \underline{c}_{N}^{c h e b},
\end{aligned}
$$


where $D_{\underline{w}_{2 N}}$ and $D_{\underline{s}_{2 N}}$ are diagonal matrices with diagonal entries $\underline{w}_{2 N}$ and $\underline{s}_{2 N}$, respectively.

The vector of Legendre coefficients $\underline{c}_{N}^{l e g}$ in (4.4) has been expressed in terms of a matrix-vector product involving $\mathbf{P}_{\mathbf{2}}\left(\underline{x}_{2 N}^{\text {cheb }}\right)^{T}$, whereas the original relation (4.1) involves the inverse $\mathbf{P}_{\mathbf{N}}\left(\underline{x}_{N}^{c h e b}\right)^{-1}$. Therefore, at the cost of doubling the size of the Legendre-Vandermonde matrices, we are able to employ the same asymptotic formula (3.3), as before. Note that the pre-multiplication by $\left[I_{N+1} \mid \mathbf{0}_{N}\right]$ in (4.4) means that only the first $N+1$ rows of $\mathbf{P}_{\mathbf{2 N}}\left(\underline{x}_{2 N}^{\text {cheb }}\right)^{T}$ are required in practice.

4.1. The transpose of the asymptotic formula. To apply the transposed Legendre-Vandermonde matrix, $\mathbf{P}_{\mathbf{2 N}}\left(\underline{x}_{2 N}^{c h e b}\right)^{T}$, we transpose the asymptotic formula for quasimatrices (3.10):

$$
\begin{array}{r}
\mathbf{P}_{\mathbf{2 N}}(\cos \theta)^{T}=\sum_{m=0}^{M-1} D_{\underline{C h}}\left(\left[0 \mid \mathbf{U}_{\mathbf{2 N}-\mathbf{1}}(\cos \theta)\right]^{T} D_{u_{m}(\theta)}+\mathbf{T}_{\mathbf{2 N}}(\cos \theta)^{T} D_{v_{m}(\theta)}\right) \\
+\mathbf{R}_{M}(\theta)^{T}
\end{array}
$$

where $x=\cos \theta$. Thus, when $x=\underline{x}_{2 N}^{\text {cheb }}=\cos \left(\underline{\theta}_{2 N}^{\text {cheb }}\right)$, the relation (4.5) expresses $\mathbf{P}_{\mathbf{2 N}}\left(\underline{x}_{2 N}^{\text {cheb }}\right)^{T}$ as a weighted sum of transposed Chebyshev-Vandermonde matrices $\mathbf{U}_{\mathbf{2 N}-\mathbf{1}}\left(\underline{x}_{2 N}^{c h e b}\right)^{T}$ and $\mathbf{T}_{\mathbf{2 N}}\left(\underline{x}_{2 N}^{c h e b}\right)^{T}$. Since we have indexed the Chebyshev points in decreasing order, the Chebyshev-Vandermonde matrix $\mathbf{T}_{\mathbf{2 N}}\left(\underline{x}_{2 N}^{c h e b}\right)$ is symmetric, i.e.,

$$
\mathbf{T}_{\mathbf{2 N}}\left(\underline{x}_{2 N}^{c h e b}\right)^{T}=\mathbf{T}_{\mathbf{2 N}}\left(\underline{x}_{2 N}^{c h e b}\right),
$$

and can be applied to a vector in the same way as before.

For $\left[0 \mid \mathbf{U}_{\mathbf{2 N}-\mathbf{1}}\left(\underline{x}_{2 N}^{\text {cheb }}\right)\right]^{T}$ we use the conversion matrix $[22]$,

$$
S_{2 N-1}=\left(\begin{array}{cccccc}
1 & 0 & -\frac{1}{2} & & & \\
& \frac{1}{2} & 0 & -\frac{1}{2} & & \\
& & \ddots & \ddots & \ddots & \\
& & & \frac{1}{2} & 0 & -\frac{1}{2} \\
& & & & \frac{1}{2} & 0 \\
& & & & & \frac{1}{2}
\end{array}\right) \in \mathbb{R}^{2 N \times 2 N}
$$

which converts Chebyshev coefficients in a series of $T_{0}, \ldots, T_{2 N-1}$ to coefficients in a series with $U_{0}, \ldots, U_{2 N-1}$ so that

$$
\mathbf{U}_{\mathbf{2 N - 1}}\left(\underline{x}_{2 N}^{\text {cheb }}\right) S_{2 N-1}=\mathbf{T}_{\mathbf{2 N - 1}}\left(\underline{x}_{2 N}^{c h e b}\right) .
$$

Using (4.6), we then have

$$
\left[\mathbf{0} \mid \mathbf{U}_{\mathbf{2 N}-\mathbf{1}}\left(\underline{x}_{2 N}^{c h e b}\right)\right]^{T}=\left[\begin{array}{c}
\mathbf{0} \\
\mathbf{U}_{\mathbf{2 N}-\mathbf{1}}\left(\underline{x}_{2 N}^{c h e b}\right)^{T}
\end{array}\right]=\left[\begin{array}{c}
\mathbf{0} \\
S_{2 N-1}^{-T} \mathbf{T}_{\mathbf{2 N}-\mathbf{1}}\left(\underline{x}_{2 N}^{c h e b}\right)
\end{array}\right] .
$$

Hence, we can apply $\left[0 \mid \mathbf{U}_{\mathbf{2 N}-\mathbf{1}}\left(\underline{x}_{2 N}^{c h e b}\right)\right]^{T}$ to a vector in $\mathcal{O}(N \log N)$ operations by using the DCT and solving a lower triangular linear system with two nonzero diagonals in $\mathcal{O}(N)$ operations. Therefore, each of the terms in the asymptotic formula can be applied in $\mathcal{O}(N \log N)$ operations, and the doubling of the Chebyshev grid means the implied constant is around a factor of two larger than the forward transform. 


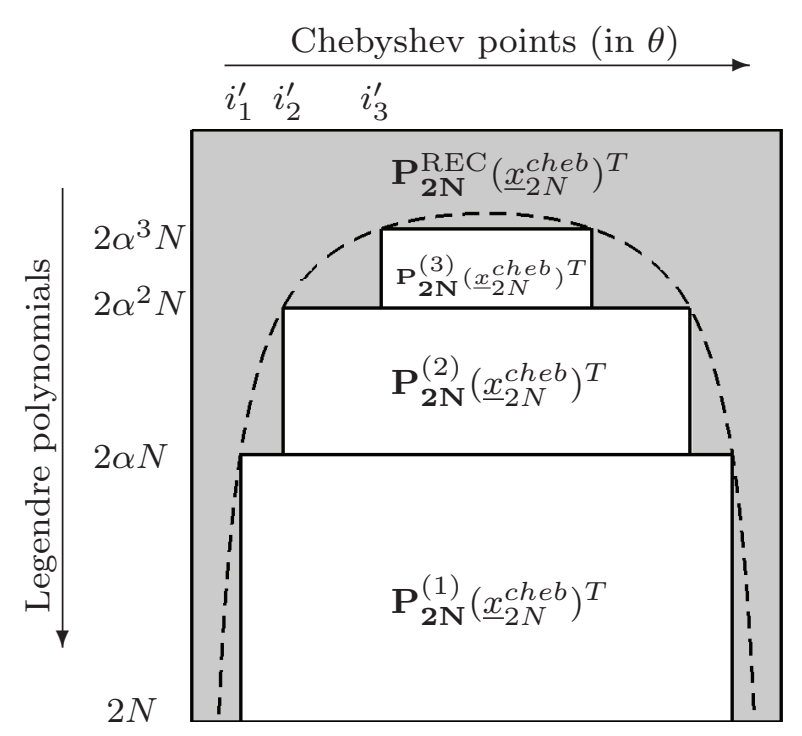

Fig. 4.1: Partitioning of the matrix $\mathbf{P}_{\mathbf{2}} \mathbf{N}\left(\underline{x}_{2 N}^{c h e b}\right)^{T}$ in the algorithm for the inverse transform. The dashed line indicates the boundary of the region in which the asymptotic formula can be employed without correction, and the gray region indicates the nonzero entries of the matrix $\mathbf{P}_{2 \mathbf{N}}^{\mathrm{REC}}\left(\underline{x}_{2 N}^{c h e b}\right)^{T}$. Again, this diagram is not drawn to scale and in practice the gray region represents a tiny proportion of the matrix $\mathbf{P}_{\mathbf{2 N}}\left(\underline{x}_{2 N}^{c h e b}\right)^{T}$.

4.2. Block partitioning for computing the inverse transform. As with the forward transform, we partition the transposed Legendre-Vandermonde matrix so that we only employ the asymptotic formula (4.5) only for entries for which it gives an accurate approximation. In fact, the block partitioning is almost identical, since the error curve (3.14) is essentially the same. In particular, we partition $\mathbf{P}_{\mathbf{2 N}}\left(\underline{x}_{2 N}^{c h e b}\right)^{T}$ so that

$$
\mathbf{P}_{\mathbf{2 N}}\left(\underline{x}_{2 N}^{\text {cheb }}\right)^{T}=\mathbf{P}_{\mathbf{2} \mathbf{N}}^{\mathrm{REC}}\left(\underline{x}_{2 N}^{\text {cheb }}\right)^{T}+\sum_{k=1}^{K} \mathbf{P}_{\mathbf{2 N}}^{(k)}\left(\underline{x}_{2 N}^{\text {cheb }}\right)^{T},
$$

which can be seen in Figure 4.1 where $i_{k}^{\prime}$ is simply (3.18) with one $N$ replaced by $2 N$.

As in Section 3.3, to balance the computation costs we require $\alpha=\mathcal{O}(1 / \log N)$, and hence $K=\mathcal{O}(\log N / \log \log N)$. To apply the matrix $\mathbf{P}_{\mathbf{2} \mathbf{N}}^{\mathrm{REC}}\left(\underline{x}_{2 N}^{\text {cheb }}\right)^{T}$ to a vector we use the three-term recurrence relation (3.16) to compute its $\mathcal{O}(K N / \alpha)$ nonzero entries. For the matrix-vector products the matrices $\mathbf{P}_{\mathbf{2}}^{(k)}\left(\underline{x}_{2 N}^{c h e b}\right)^{T}$ for $k=1, \ldots, K$, we use the transposed asymptotic formula (4.5) evaluated by using the DCT and the relationship (4.7). Hence, in total, the numerically stable algorithm described for the inverse transform requires $\mathcal{O}\left(N(\log N)^{2} / \log \log N\right)$ operations to convert $N$ Chebyshev coefficients to Legendre coefficients.

5. Numerical results. Here, we no longer consider the unstable algorithm for the forward transform, described in Section 3.2, and instead concentrate on the algorithms that we advocate for the forward and inverse transform. All numerical experiments were performed on a single core of a 2011 1.8GHz Intel Core i7 MacBook Air with Matlab 2013a. Execution times should be considered as approximate. The accuracy results are determined by comparing to an extended precision multiplication of the vector of coefficients by the transformation matrices $L^{n}$ and $M^{n}$ from [2]. For timing comparisons, we compare against the direct multiplication of a vector by $\mathbf{P}_{N}\left(\underline{x}_{N}^{c h e b}\right)$ computed in MATLAB via the three-term recurrence relation.

5.1. Numerical results for the forward transform. In our implementation we use $M=10$ for all $N$, though the efficiency of the algorithm for the forward transform is not particularly sensitive to the choice of $M$ (see Figure 5.1 (left)). For 

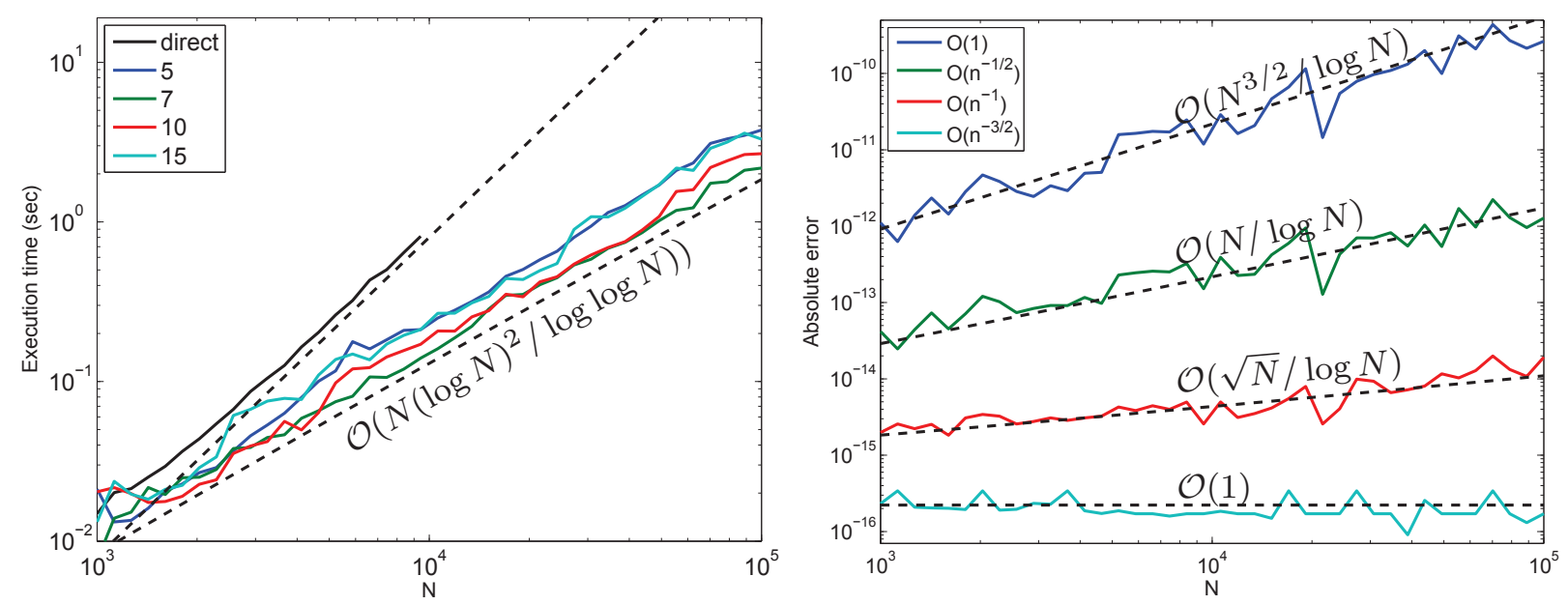

Fig. 5.1: Left: Execution times for the forward transform for $10^{3} \leq N \leq 10^{5}$ and $M=5,7,10,15$ in Matlab. Right: The absolute error in the Chebyshev coefficients after converting $N+1$ Legendre coefficients to Chebyshev with different decay rates: no decay (blue), $N^{-1 / 2}$ (green), $N^{-1}$ (red), and $N^{-3 / 2}$ (cyan).

$M=10$ we find our algorithm is faster than the direct $O\left(N^{2}\right)$ computation when $N \geq 512$, and that $N=10^{6}$ takes 31.2 seconds.

The dominating computational cost of our algorithm is in computing the DCT. Unfortunately, MATLAB does not natively support a DCT command ${ }^{4}$, but we can achieve the same transform via a FFT as follows [11,34]:

function $\mathrm{v}=\operatorname{det} 1$ (c)

$\%$ DCT1 Compute a (scaled) DCT of type 1 using the FFT.

$\% \operatorname{DCT} 1(\mathrm{C})=\mathrm{T}_{-} \mathrm{N}\left(\mathrm{X}_{-} \mathrm{N}\right) * \mathrm{C}$, where $\mathrm{X}_{-} \mathrm{N}=\cos (\mathrm{pi} *(0: \mathrm{N})) / \mathrm{N}$ and $\mathrm{T}_{-} \mathrm{N}(\mathrm{X})=\left[\mathrm{T}_{-} 0\right.$,

$\left.\% \mathrm{~T}_{-} 1, \ldots, \mathrm{T}_{-} \mathrm{N}\right](\mathrm{X})$. $\mathrm{T}_{-} \mathrm{k}$ is the kth 1st-kind Chebyshev polynomial.

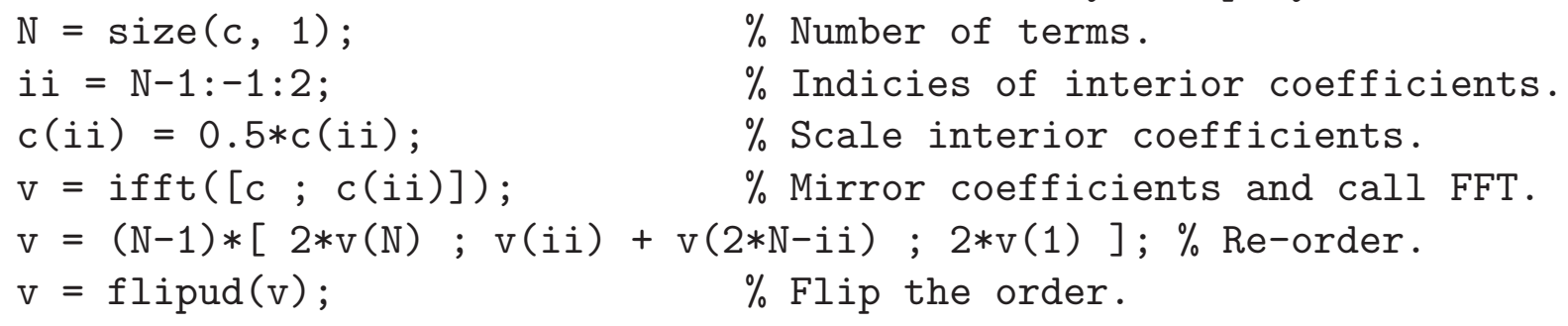

However, the vector is doubled in length before applying the FFT and this means that this is twice as expensive as a DCT. We expect that the execution times of our algorithm would improve by nearly a factor of two if MATLAB allowed direct access to the FFTW DCT routines [9]. In Figure 5.1 (left) we show the execution times of the forward transform for $M=5,7,10,15$ and $10^{3} \leq N \leq N^{5}$.

To get an idea of the accuracy we can expect in the forward transform, suppose the entries of the vector $\underline{c}_{N}^{l e g}$ decay like $n^{-r}$, i.e., $\left(\underline{c}_{N}^{l e g}\right)_{n}=\mathcal{O}\left(n^{-r}\right)$, and define $D_{r}=$ $\operatorname{diag}\left(1^{-r}, \ldots, N^{-r}\right)$. Then we have

$$
\underline{c}_{N}^{l e g}=D_{r} \underline{\hat{c}}_{N}^{l e g}
$$

where the vector $\underline{\hat{c}}_{N}^{\text {leg }}$ has no decay and hence,

$$
\left\|\mathbf{P}_{\mathbf{N}}\left(\underline{x}_{N}^{c h e b}\right) \underline{c}_{N}^{l e g}\right\|_{\infty} \leq\left\|\mathbf{P}_{\mathbf{N}}\left(\underline{x}_{N}^{c h e b}\right) D_{r}\right\|_{\infty}\left\|\underline{\hat{c}}_{N}^{l e g}\right\|_{\infty}
$$

${ }^{4}$ Note that the Signal Process Toolbox in MATLAB does supply a DCT command, but this utilizes the FFT in a similar way to the dct1 code given above. 

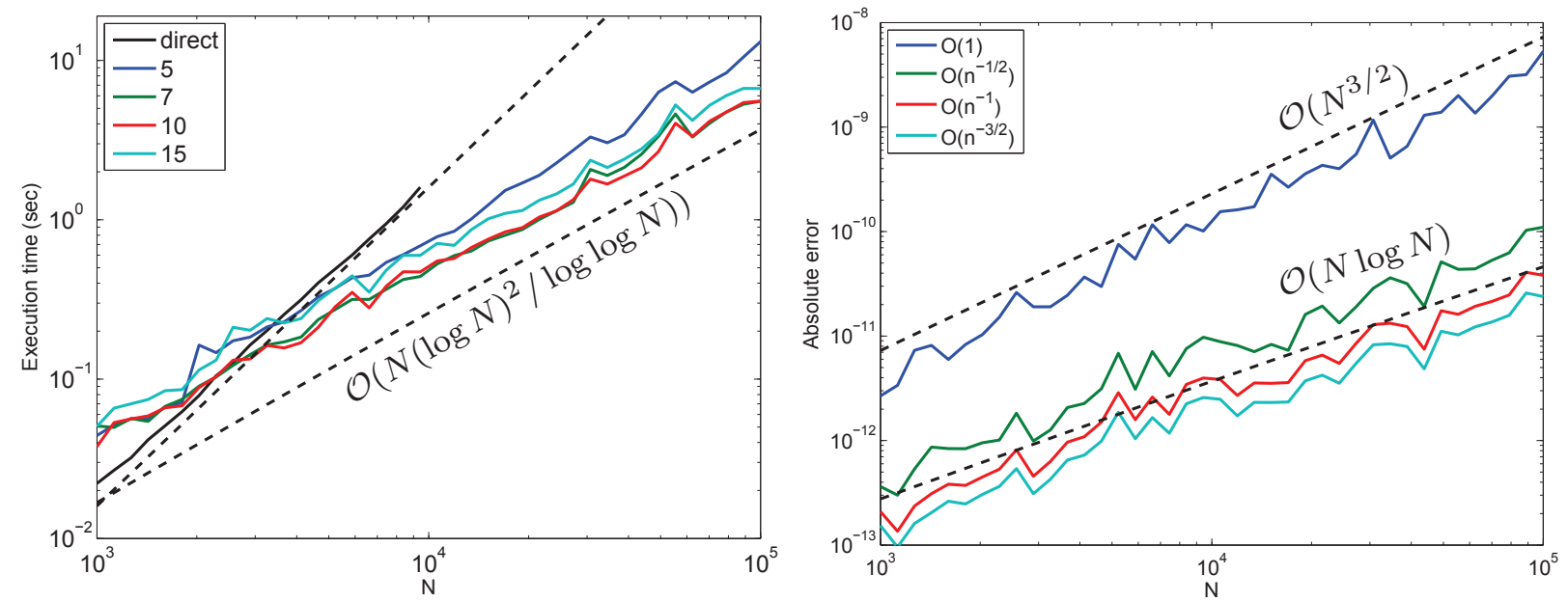

Fig. 5.2: Left: Execution times for the inverse transform for $10^{3} \leq N \leq 10^{5}$ and $M=5,7,10,15$ in Matlab. Right: The absolute error in the $N$ computed Legendre coefficients after converting Chebyshev coefficients with different decay rates: no decay (blue), $n^{-1 / 2}$ (green), $n^{-1}$ (red), and $n^{-3 / 2}$ (cyan).

Since $\max _{x \in[-1,1]} P_{n}(x)=P_{n}(1)=1$, the infinity norm of the matrix $\mathbf{P}_{\mathbf{N}}\left(\underline{x}_{N}^{c h e b}\right) D_{r}$ is the absolute sum of the first row, and hence,

$$
\left\|\mathbf{P}_{\mathbf{N}}\left(\underline{x}_{N}^{\text {cheb }}\right) D_{r}\right\|_{\infty}=\sum_{n=1}^{N} n^{-r}=H_{N, r}= \begin{cases}N, & r=0, \\ \mathcal{O}(\sqrt{N}), & r=1 / 2, \\ \mathcal{O}(\log N), & r=1, \\ \mathcal{O}(1), & r>1,\end{cases}
$$

where $H_{N, r}$ is the generalized harmonic number [3, Theorem 3.2]. Therefore, we expect the absolute error of the forward transform to grow something like $H_{N, r}$.

To investigate the actual error we observe in computing the forward transform we take random vectors ${ }^{5}$ of Legendre coefficients that have entries decaying like $n^{-r}$ with $r=0,1 / 2,1,3 / 2$. Figure 5.1 (right) shows the maximum absolute error in the computed Chebyshev coefficients. We observe an error growth of $\mathcal{O}\left(N^{3 / 2-r} / \log N\right)$ for $r=0,1 / 2,1$ in the computed vector $\underline{c}_{N}^{c h e b}$ and no error growth for $r=3 / 2$. For $r=0,1 / 2,1$, this observed error growth seems to be $\sqrt{N} / \log N$ times worse than $H_{N, r}$, and we cannot explain this mysterious factor. However, for suitably decaying vectors $\underline{c}_{N}^{l e g}$, i.e., entries that decay like $N^{-3 / 2}$ or faster, the error in $\mathbf{P}_{\mathbf{N}}\left(\underline{x}_{N}^{c h e b}\right) \underline{c}_{N}^{l e g}$ remains bounded with $N$. Often, in practice, a Legendre expansion approximates a smooth function and hence the coefficients decay sufficiently.

5.2. Numerical results for the inverse transform. Our implementation of the inverse transform also uses 10 terms in the asymptotic formula (4.5), but as before the the efficiency of the algorithm is not particularly sensitive to the value of $M$ (Figure 5.2 (left)). The cost of the inverse transform is approximately twice that of the forward transform because the algorithm for the inverse evaluates DCTs on vectors of twice the length.

In Figure 5.2 (right) we repeat the same accuracy experiment for the inverse transform. This time we observe an error growth of $\mathcal{O}\left(N^{3 / 2}\right)$ for $r=0$ and $\mathcal{O}(N \log N)$ for $r=1 / 2,1,3 / 2$. An error growth of $\mathcal{O}(N)$ for any $r \geq 1 / 2$ is due to the orthogonalization scaling factor, $\left\|P_{n}\right\|_{2}^{-2}$, that appears in the integral definition (4.2)

${ }^{5}$ Vectors are generated with the MATLAB command randn with the random number generator mt19937ar and rng(1), and then introduce decay by scaling the $n$th entry by $n^{-r}$. 
of the Legendre coefficients. Conventionally, Legendre polynomials are scaled so that $P_{n}(1)=1$ for all $n$, and for this choice $\left\|P_{n}\right\|_{2}^{-2}=n+1 / 2$. Our algorithm computes the coefficients in the orthonormal Legendre basis to essentially machine precision, but to convert them to the standard Legendre basis the $n$th coefficient is multiplied by $n+1 / 2$. In addition, we again observe a mysterious log factor that we cannot explain.

6. Extensions and conclusion. We have presented a fast Chebyshev-Legendre transform based on the asymptotic formula (3.3), and here we give possible extensions to other related fast transforms.

Fast evaluation of Legendre expansions. The forward transform converts Legendre coefficients to Chebyshev coefficients, and as an intermediary step of the transform the Legendre expansion of degree $N(1.2)$ is evaluated at the vector of Chebyshev points $\underline{x}_{N}^{c h e b}$. More precisely, $\underline{x}_{N}^{\text {cheb }}$ is the vector of Chebyshev points of the second kind. This immediately gives a fast algorithm for evaluating a Legendre expansion of degree $N$ at $\underline{x}_{N}^{\text {cheb }}$ in $\mathcal{O}\left(N(\log N)^{2} / \log \log N\right)$ operations. A similar fast transform can be derived that evaluates a Legendre expansion at any other set of Chebyshev points:

First kind: $x_{k}=\cos \left(\frac{\left(k+\frac{1}{2}\right) \pi}{N+1}\right), 0 \leq k \leq N$, the roots of $T_{N+1}$;

Second kind: $x_{k}=\cos \left(\frac{k \pi}{N}\right), 0 \leq k \leq N$, the roots of $U_{N-1}$ and \pm 1 ;

Third kind: $x_{k}=\cos \left(\frac{k \pi}{N+\frac{1}{2}}\right), 0 \leq k \leq N$, the roots of $V_{N}$ and 1 (see [21]);

Fourth kind: $x_{k}=\cos \left(\frac{\left(k+\frac{1}{2}\right) \pi}{N+\frac{1}{2}}\right), 0 \leq k \leq N$, the roots of $W_{N}$ and -1 (see [21]).

These algorithms would still employ a DCT, but it would be of a different type: DCTI for second kind, DCT-III for first kind, DCT-V for third kind, and DCT-VII for fourth kind (see [37] for more details).

Fast Chebyshev-Jacobi transform. Legendre polynomials are a special case of Jacobi polynomials, and Hahn $[13,21]$ gives a more general asymptotic formula that remains remarkably similar to (3.3). We expect that this asymptotic formula also leads to a fast Chebyshev-Jacobi transform with approximately the same methodology. However, we have not yet been able to find an accompanying explicit error formula, which is useful for deriving the details of the algorithm.

Furthermore, although the Clenshaw-Curtis-Jacobi weights required for the quadrature rule in (4.3) can be computed in $O(N \log N)$ operations when the Jacobi parameters $\alpha$ and $\beta$ are equal [28] (i.e., for so-called Gegenbauer or ultrapsherical polynomials), it is still an open problem for $\alpha \neq \beta$. Since the asymptotic formula for the ultraspherical case is simpler than for general Jacobi polynomials [32, (8.21.14)], this would be a sensible next step.

Fast spherical harmonic transform. The spherical harmonic expansion of a function takes the form

$$
f(\theta, \phi)=\sum_{n=0}^{\infty} \sum_{m=-n}^{n} \alpha_{n}^{m} P_{n}^{|m|}(\cos \theta) e^{i m \phi},
$$

where $(\theta, \phi)$ are spherical coordinates parameterizing the surface of the sphere embedded in $\mathbb{R}^{3}$ and $P_{n}^{|m|}$ is the associated Legendre polynomial of degree $n$ and order $|m|[26]$. There are many algorithms for the fast spherical harmonic transform $[18,26,31]$, but it may also be possible to derive an algorithm that has a similar 
flavor to this paper. It would be interesting to consider the advantages, if any, of a fast algorithm for this transform based on an asymptotic formula for $P_{n}^{|m|}$, and we leave this for future work.

Conclusion. We have presented an $\mathcal{O}\left(N(\log N)^{2} / \log \log N\right)$ algorithm for the Chebyshev-Legendre transform, which is faster than the direct approach for $N \geq$ 512. We block partitioned the Legendre-Vandermonde-like matrix to ensure that the asymptotic formula was evaluated only when it is valid, thus ensuring stability of the algorithm, and the use of DCTs allowed fast evaluation. If the coefficients in a truncated series expansion decay faster than $n^{-3 / 2}$, then the forward transform has no error growth with $N$ and the inverse transform has an error growth of $\mathcal{O}(N \log N)$. Our publicly available MATLAB implementation can convert between as many as one million Legendre coefficients and Chebyshev coefficients with high accuracy.

Acknowledgments. We would like to thank Nick Trefethen for his personal, intellectual, and financial support throughout this work, and Anthony Austin for his assistance in translating a significant portion of the Japanese paper [19]. We would also like to thank André Weideman, Sheehan Olver, and the Chebfun team for stimulating discussions, and are grateful to Reiji Suda for privately communicating an unpublished manuscript. The first author was supported by The MathWorks, Inc., and King Abdullah University of Science and Technology (KAUST), Award No. KUK-C1-013-04). The second author is supported by EPSRC grant EP/P505666/1 and the European Research Council under the European Union's Seventh Framework Programme (FP7/2007-2013)/ERC grant agreement no. 291068.

\section{REFERENCES}

[1] N. Ahmed, T. Natarajan, And K. R. Rao, Discrete cosine transform, IEEE Trans. Comput., 100 (1974), pp. 90-93.

[2] B. K. Alpert And V. Rokhlin, A fast algorithm for the evaluation of Legendre expansions, SIAM J. Sci. Stat. Comput., 12 (1991), pp. 158-179.

[3] T. M. Apostol, Introduction to Analytic Number Theory, Springer International Student Edition, Undergraduate Texts in Mathematics, Springer, 1976.

[4] K. Atkinson And W. Han, Spherical Harmonics and Approximations on the Unit Sphere: An Introduction, Volume 2044 of Lecture Notes in Mathematics, Springer, 2012.

[5] I. Bogaert, B. Michiels AND J. Fostier, $\mathcal{O}(1)$ computation of Legendre polynomials and Gauss-Legendre nodes and weights for parallel computing, J. Sci. Comput., 34 (2012), pp. C83-C101.

[6] J. P. Boyd, Chebyshev and Fourier Spectral Methods, Dover Publications, 2001.

[7] E. De Micheli And G. A. Viano, The expansion in Gegenbauer polynomials: A simple method for the fast computation of the Gegenbauer coefficients, J. Comput. Phys., 239 (2013), pp. 112-122.

[8] W. S. Don And D. GotTlieb, The Chebyshev-Legendre method: Implementing Legendre methods on Chebyshev points, SIAM J. Numer. Anal., 31 (1994), pp. 1519-1534.

[9] M. Frigo And S. G. Johnson, The Design and Implementation of FFTW3, Proceedings of the IEEE, Special issue on "Program Generation, Optimization, and Platform Adaptation", 93 (2005), pp. 216-231.

[10] N. Gallagher, G. Wise, And J. Allen, A novel approach for the computation of Legendre polynomial expansions, IEEE Trans. Acoustic, Speech and Signal Processing, 26 (1978), pp. 105-106.

[11] W. M. Gentleman, Implementing Clenshaw-Curtis quadrature II: Computing the cosine transformation, Communications of the ACM, 15 (1972), pp. 343-346.

[12] M. GU AND S. C. EISEnstat, A divide-and-conquer algorithm for the symmetric tridiagonal eigenproblem, SIAM J. Matrix Anal. Appl., 16 (1995), pp. 172-191.

[13] E. Hahn, Asymptotik bei Jacobi-Polynomen und Jacobi-Funktionen, Mathematische Zeitschrift, 171 (1980), pp. 201-226. 
$\%$ Use direct approach if $\mathrm{N}$ is small:

if $(M==0|| N<513|| K==0)$, c_cheb = leg2cheb_direct(c_leg); return, end

$\mathrm{t}=\mathrm{pi} *(0: \mathrm{N}), / \mathrm{N}$

$\mathrm{nM}=\operatorname{ceil}\left(\mathrm{aM} .^{-}(\mathrm{K}-1:-1: 0) * \mathrm{~N}\right)$;

$j \mathrm{~K}=\operatorname{zeros}(\mathrm{K}, 2)$;

for $k=1: K$

$\operatorname{tmp}=f$ ind $(t>=\operatorname{asin}(n M 0 . / n M(k)), 1)-4 ; \%$ Where curve intersects $\operatorname{aM}^{\wedge} k * N$.

$j K(k,:)=[t m p+1, N+1-t m p]$;
$\%$ Theta variable.

$\%$ n_M for each block.

$\%$ Block locations in theta.

$\%$ Collect indicies.

end

$\% \% \% \% \% \% \% \% \% \% \% \% \% \% \% \% \% \% \%$ Recurrence / boundary region $\% \% \% \% \% \% \% \% \% \% \% \% \% \% \% \% \% \% \% \% \% \% \% \% \% \% \%$ v_rec $=\operatorname{zeros}(\mathrm{N}+1,1)$;

$j K 2=[j K(1,2)+1, N+1 ; j K(1: K-1,:) ; 1, N+1]$;

for $\mathrm{k}=1: \mathrm{K} \%$ Loop over the partitions:

$j_{-}$bdy $=[j K 2(k+1,1): j K 2(k, 1)-1, j K 2(k, 2)+1: j K 2(k+1,2)]$;

$\mathrm{x}_{-} \mathrm{bdy}=\cos \left(\mathrm{t}\left(\mathrm{j}_{-} \mathrm{bdy}\right)\right) ; \quad \%$ Boundary indicies.

tmp $=c_{-} \operatorname{leg}(1)+c_{-} \operatorname{leg}(2) * x_{-} b d y ; \quad \%$ Entries of mat-vec result.

$\operatorname{Pm} 2=1 ; \operatorname{Pm} 1=x_{-}$bdy; $\quad \%$ Initialise recurrence.

for $\mathrm{n}=1: \mathrm{nM}(\mathrm{k})-1 \%$ Recurrence:

$\mathrm{P}=(2-1 /(\mathrm{n}+1)) * \operatorname{Pm} 1 . * \mathrm{x}_{-} \mathrm{bdy}-\mathrm{n} /(\mathrm{n}+1) * \operatorname{Pm} 2$;

$\mathrm{Pm} 2=\operatorname{Pm} 1 ; \operatorname{Pm} 1=\mathrm{P}$;

end

tmp $=$ tmp $+c_{-}$leg $(n+2) * P ; \quad \%$ Update local LHS.

v_rec $\left(j_{\text {_bdy }}\right)=\mathrm{v}_{\text {_rec }}\left(j_{\text {_bdy }}\right)+\operatorname{tmp} ; \quad \%$ Global correction LHS.

end

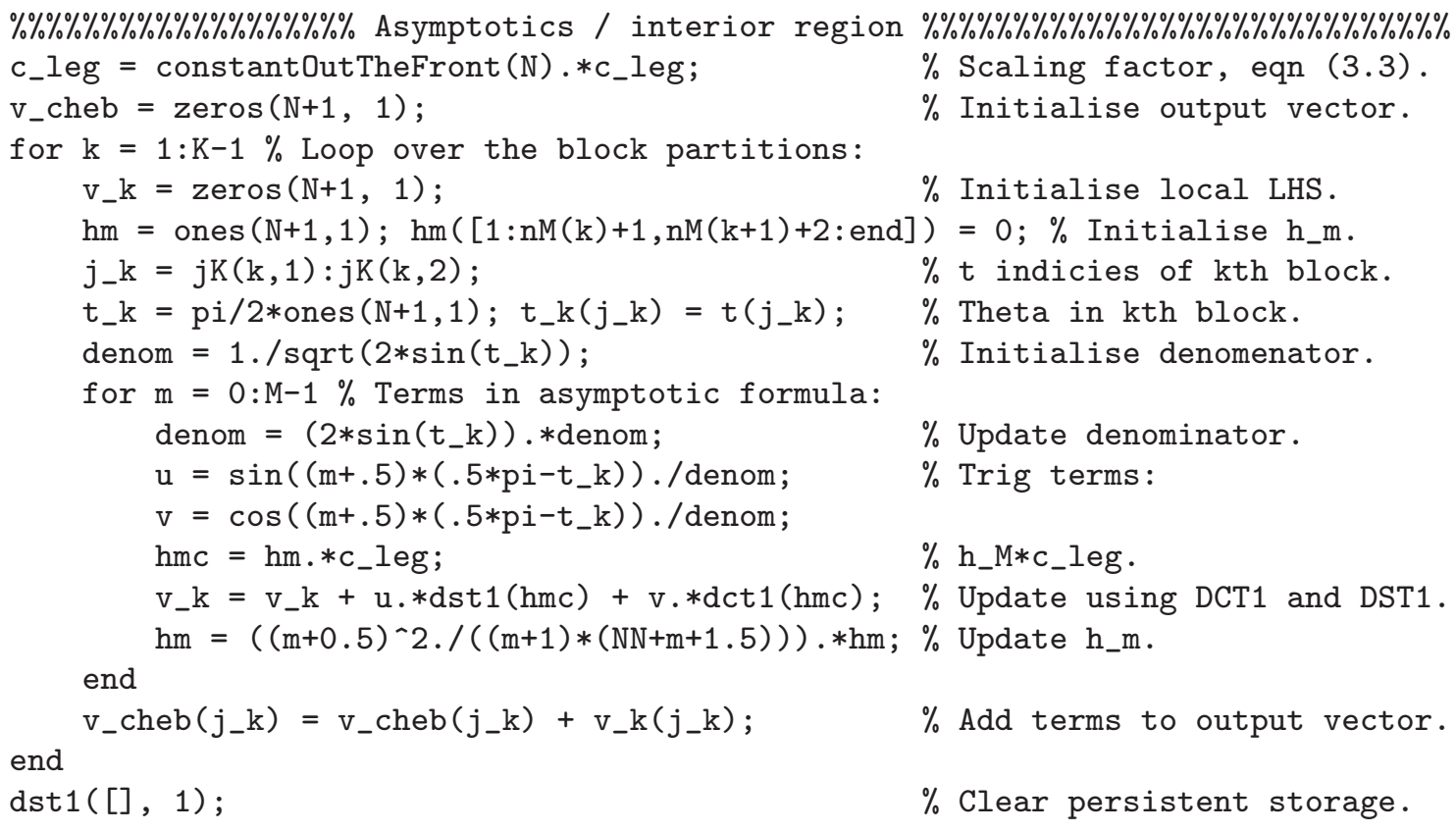

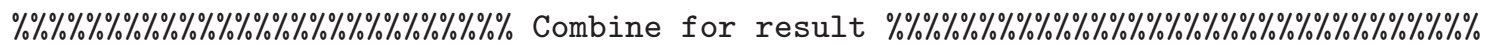
$\mathrm{v}_{\text {_cheb }}=\mathrm{v}_{-}$cheb $+\mathrm{v}_{-}$rec; $\quad \%$ Values on Chebyshev grid.

$c_{-}$cheb = idct1 $\left(v_{-}\right.$cheb); $\quad \%$ Chebyshev coeffs.

end

function $\mathrm{C}=$ constantOutTheFront(N) \% (See, Hale and Townsend [13])

$\%$ CONSTANTOUTTHEFRONT $(N)$ returns $\operatorname{sqrt}(4 / \mathrm{pi}) * \operatorname{gamma}((0: N)+1) / \operatorname{gamma}((0: N)+3 / 2))$

$\%$ Initialise:

$\mathrm{NN}=(0: \mathrm{N})^{\prime} ;$

NN(1) $=1 ; \%$ Set the first value different from 0 to avoid complications.

$\mathrm{ds}=-1 / 8 . / \mathrm{NN} ; \mathrm{s}=\mathrm{ds} ; \mathrm{j}=1 ; \mathrm{ds}(1)=1 ;$

while ( $\operatorname{norm}(\mathrm{ds}(10:$ end $) . / \mathrm{s}(10:$ end $)$, inf $)>$ eps/100 ) 


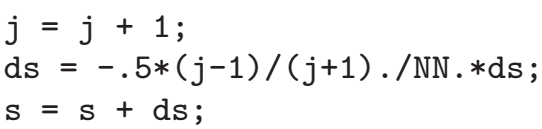

end

$\mathrm{NN}(1)=0 ; \%$ Reset the first value.

$\mathrm{p} 2=\exp (\mathrm{s}) \cdot * \operatorname{sqrt}(4 . /(\mathrm{NN}+.5) / \mathrm{pi}) ;$

$\%$ Stirling's series:

$g=\left[\begin{array}{lllllll}1 & 1 / 12 & 1 / 288 & -139 / 51840 & -571 / 2488320 & 163879 / 209018880 & \ldots\end{array}\right.$

$5246819 / 75246796800-534703531 / 902961561600 \ldots$

-4483131259/86684309913600 432261921612371/514904800886784000];

$\mathrm{eN}=\operatorname{ones}(\mathrm{N}+1,1) ; \mathrm{e} 9=\operatorname{ones}(1,9)$;

ff1 = sum(bsxfun(@times, g, [eN, cumprod(bsxfun(@rdivide, e9, NN),2)]), 2);

ff2 = sum(bsxfun(@times, g, [eN, cumprod(bsxfun(@rdivide, e9, NN+.5),2)]), 2);

$C=p 2 . * f f 1 . / f f 2$;

$\%$ Use direct evaluation for the small values:

$\mathrm{C}(1: 10)=\operatorname{sqrt}(4 / \mathrm{pi}) * \operatorname{gamma}((0: 9)+1) \cdot / \operatorname{gamma}((0: 9)+3 / 2) ;$

end

function $\mathrm{v}=\operatorname{dst} 1$ (c, flag) $\%$ \# ok<INUSD>

$\%$ DST1 Discrete sine transform of type 1.

$\% \operatorname{DST1}(\mathrm{C})$ returns $\operatorname{diag}\left(\sin \left(\mathrm{T}_{-} \mathrm{N}\right)\right) * \mathrm{U}_{-} \mathrm{N}(\mathrm{X}) * \mathrm{C}$ where $\mathrm{T}_{-} \mathrm{N}(\mathrm{k}, 1)=\mathrm{pi} *(\mathrm{k}-1) / \mathrm{N}, \mathrm{k}=$

$\% 1: N+1, X_{-} N=\cos \left(T_{-} N\right)$ and $U_{-} N(X)=\left[U_{-} 0, U_{-} 1, \ldots, U_{-} N\right](X)$ where U_k is the

$\%$ kth 2nd-kind Chebyshev polynomial.

persistent Smat sint $\quad \%$ The same for each partition.

if ( nargin == 2 ), Smat = [] ; return, end \% Clear persistent variables.

if ( isempty(Smat) ) \% Construct conversion matrix:

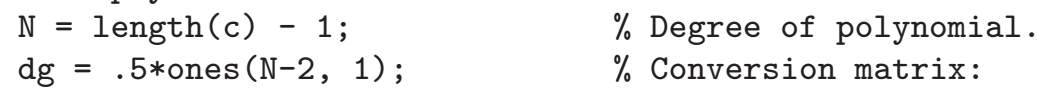

end

$\mathrm{v}=\operatorname{sint} . * \operatorname{dct} 1([\operatorname{Smat} \backslash c(2:$ end $) ; 0]) ; \%$ Scaled DCT.

end

function $c=\operatorname{idct1}(v)$

\%IDCT1 Convert values on a Cheb grid to Cheb coefficients (inverse DCT1).

$\% \operatorname{IDCT1}(\mathrm{V})$ returns $\mathrm{T}_{-} \mathrm{N}\left(\mathrm{X}_{-} \mathrm{N}\right) \backslash \mathrm{V}$, where $\mathrm{X}_{-} \mathrm{N}=\cos (\mathrm{pi} *(0: \mathrm{N})) / \mathrm{N}$ and $\mathrm{T}_{-} \mathrm{N}(\mathrm{X})=\left[\mathrm{T}_{-} 0\right.$,

$\left.\% \mathrm{~T}_{-} 1, \ldots, \mathrm{T}_{-} \mathrm{N}\right](\mathrm{X})$ where $\mathrm{T}_{-} \mathrm{k}$ is the kth 1st-kind Chebyshev polynomial.

$\mathrm{N}=\operatorname{size}(\mathrm{v}, 1) ;$

$\mathrm{c}=\operatorname{fft}([\mathrm{v} ; \mathrm{v}(\mathrm{N}-1:-1: 2)]) /(2 * \mathrm{~N}-2) ; \%$ Laurent fold in columns and call FFT.

$c=c(1: N) ; \quad \%$ Extract the first $N$ terms.

if $(\mathrm{N}>2), c(2: \mathrm{N}-1)=2 * c(2: \mathrm{N}-1)$; end $\%$ Scale interior coefficients.

if isreal(v), $c=\operatorname{real}(\mathrm{c})$; end $\%$ Ensure a real output.

end

function $c_{-}$cheb = leg2cheb_direct (c_leg)

\%LEG2CHEB_DIRECT Convert Leg to Cheb coeffs using the 3-term recurrence.

$\mathrm{N}=$ length (c_leg) - 1; $\quad \%$ Degree of polynomial.

if $(\mathrm{N}<=0), c_{-}$cheb $=c_{-}$leg; return, end $\%$ Trivial case.

$\mathrm{x}=\cos \left(\mathrm{pi} *(0: \mathrm{N})^{\prime} / \mathrm{N}\right) ; \quad \%$ Chebyshev grid (reversed order).

$\%$ Make the Legendre-Chebyshev Vandemonde matrix:

$\mathrm{Pm} 2=1 ; \operatorname{Pm} 1=\mathrm{x} ; \quad \%$ Initialise.

$\mathrm{L}=\operatorname{zeros}(\mathrm{N}+1, \mathrm{~N}+1) ; \quad \%$ Vandermonde matrix.

$\mathrm{L}(:, 1: 2)=[1+0 * \mathrm{x}, \mathrm{x}] ; \quad \% \mathrm{P}_{-} 0$ and $\mathrm{P}_{-} 1$.

for $\mathrm{n}=1: \mathrm{N}-1 \%$ Recurrence relation:

$\mathrm{P}=(2-1 /(\mathrm{n}+1)) * \operatorname{Pm} 1 . * \mathrm{x}-\mathrm{n} /(\mathrm{n}+1) * \operatorname{Pm} 2 ;$

$\mathrm{Pm} 2=\mathrm{Pm} 1 ; \mathrm{Pm} 1=\mathrm{P}$;

$\mathrm{L}(:, 2+\mathrm{n})=\mathrm{P}$;

end

$v_{\text {_cheb }}=$ L*c_leg;

$c_{-}$cheb $=\operatorname{idct1}\left(v_{-}\right.$cheb) ;

$\%$ Values on Chebyshev grid.

end

$\%$ Chebyshev coefficients. 